\title{
Discovery of Siderophore and Metallophore Production in the Aerobic Anoxygenic Phototrophs
}

\author{
Steven B. Kuzyk, Elizabeth Hughes and Vladimir Yurkov * \\ Department of Microbiology, University of Manitoba, Winnipeg, MB R3T 2N2, Canada; \\ umkuzyks@myumanitoba.ca (S.B.K.); elizabeth.hughes@umanitoba.ca (E.H.) \\ * Correspondence: Vladimir.Yurkov@umanitoba.ca
}

check for updates

Citation: Kuzyk, S.B.; Hughes, E.; Yurkov, V. Discovery of Siderophore and Metallophore Production in the Aerobic Anoxygenic Phototrophs. Microorganisms 2021, 9, 959.

https://doi.org/10.3390/

microorganisms 9050959

Academic Editor: Johannes F. Imhoff

Received: 22 March 2021

Accepted: 28 April 2021

Published: 29 April 2021

Publisher's Note: MDPI stays neutral with regard to jurisdictional claims in published maps and institutional affiliations.

Copyright: (C) 2021 by the authors. Licensee MDPI, Basel, Switzerland. This article is an open access article distributed under the terms and conditions of the Creative Commons Attribution (CC BY) license (https:/ / creativecommons.org/licenses/by/ $4.0 /)$.

\begin{abstract}
Aerobic anoxygenic phototrophs have been isolated from a rich variety of environments including marine ecosystems, freshwater and meromictic lakes, hypersaline springs, and biological soil crusts, all in the hopes of understanding their ecological niche. Over 100 isolates were chosen for this study, representing 44 species from 27 genera. Interactions with $\mathrm{Fe}^{3+}$ and other metal(loid) cations such as $\mathrm{Mg}^{2+}, \mathrm{V}^{3+}, \mathrm{Mn}^{2+}, \mathrm{Co}^{2+}, \mathrm{Ni}^{2+}, \mathrm{Cu}^{2+}, \mathrm{Zn}^{2+}, \mathrm{Se}^{4+}$ and $\mathrm{Te}^{2+}$ were tested using a chromeazurol S assay to detect siderophore or metallophore production, respectively. Representatives from 20 species in 14 genera of $\alpha$-Proteobacteria, or 30\% of strains, produced highly diffusible siderophores that could bind one or more metal(loid)s, with activity strength as follows: $\mathrm{Fe}>\mathrm{Zn}>\mathrm{V}>\mathrm{Te}>\mathrm{Cu}>\mathrm{Mn}>\mathrm{Mg}>$ $\mathrm{Se}>\mathrm{Ni}>\mathrm{Co}$. In addition, $\gamma$-proteobacterial Chromocurvus halotolerans, strain EG19 excreted a brown compound into growth medium, which was purified and confirmed to act as a siderophore. It had an approximate size of $\sim 341 \mathrm{Da}$ and drew similarities to the siderophore rhodotorulic acid, a member of the hydroxamate group, previously found only among yeasts. This study is the first to discover siderophore production to be widespread among the aerobic anoxygenic phototrophs, which may be another key method of metal(loid) chelation and potential detoxification within their environments.
\end{abstract}

Keywords: aerobic anoxygenic phototrophs; siderophore; metallophore; CAS assay; Chromocurvus halotolerans strain EG19

\section{Introduction}

Iron $(\mathrm{Fe})$ is an essential element for life. In microorganisms, it is used as a co-factor for enzymatic processes, such as in electron transfer during respiration and photosynthesis, nucleic acid or chlorophyll synthesis, nitrate reduction, nitrogen fixation and detoxification of oxygen radicals [1-3]. The use of Fe in bacteriochlorophyll (BChl) synthesis and the process of photophosphorylation makes it particularly important to phototrophic organisms. Aerobic anoxygenic phototrophs (AAP) are one such physiological group that uses photosynthesis in oxic conditions as an additional energy source to respiration [4]. They make up a significant proportion of many bacterial communities from a host of environments [5], and therefore likely require a substantial Fe uptake. In addition, Fe may be crucial to protect the cells from oxidative stress due to singlet oxygen formation during BChl $a$ synthesis. While necessary for metabolism, biologically active Fe is typically quite sparse in nature as its soluble level is very low at soil and water surfaces [2]. In response to this limitation, both bacteria and fungi have developed siderophores to compete for the available Fe [6]. Siderophores, aptly named from the Greek root representing "iron-bearing" [7], are low weight molecules (no more than 1500-2000 Da and generally lower than $1000 \mathrm{Da}$ ) with a high-affinity for Fe [2,3,8-10]. The molecules are often short polypeptides with modified or D-amino acids $[2,9,11]$. They can also be made from dicarboxylic acids and diamine or amino alcohols, linked by amide and ester bonds. These building blocks retain some characteristics of amino acids [2]. Siderophores can be classified into two main functional groups. The first is the hydroxamate group, which involves hydroxamic acid and is produced by both fungi and bacteria. Second is the catechol group, compounds of which 
contain catechol rings and are only produced by bacteria $[6,9,10]$. Smaller groups such as the hydroxyacids and the $\alpha$-hydroxy carboxylates are only rarely used by bacteria $[9,10]$. Usually, siderophores are synthesized and secreted by cells under iron-deplete growth conditions [8]. Once bound to iron, the complex is taken up by the cells in a substrate specific process [2].

While the main purpose of siderophores is Fe acquisition, they may also play some additional roles. In Pseudomonas aeruginosa, pyoverdine controls virulence factor production [12]. Escherichia coli can be protected from oxidative stress by the catechol type siderophore enterobactin [13]. Pyochelin in P. aeruginosa has a toxic effect on eukaryotic cells, possibly aiding in the bacterium's virulence [13]. Watasemycins, sideromycins, oxachelin and fusigen have antibacterial activity, which may aid in community competition by preventing other populations from growing [13]. Additionally, siderophores have been shown to bind more than one metal [3,14], including some that have higher affinity for $\mathrm{Cu}$ or $\mathrm{Zn}$ rather than Fe $[15,16]$. This broad-spectrum activity has required the classification "metallophore" [17,18], which is a term used for secondary metabolites capable of binding a range of metal(loid) cations. When a metallophore would have a specific metal affinity, it would have a sub-categorical name, where siderophore is for Fe-binding, chalkophore for $\mathrm{Cu}$, or zincophore for $\mathrm{Zn}$, all named when discovered.

This concept, of metallophores capable of capturing multiple metal cations has implicated usefulness in toxic heavy metal tolerances. Particularly in extreme environments, where metals can be present at elevated concentrations that inhibit a variety of life $[19,20]$. Many metal ions can diffuse freely through the cellular membrane, which is inhibited if the metal is bound to a siderophore that is too large to move without active transport. Furthermore, membrane receptors specific to siderophore-iron complexes can differentiate between those containing substitute cations, causing the cell to reject the alternative metalcontaining siderophore. The reduction of free metal concentrations in proximity of the bacterium and decreased passive diffusion of unwanted metals into the cell will lower their overall toxic effect [3]. This could be a compelling concept, as AAP possess very high levels of resistance to toxic heavy metal(loid) oxides [21]. While internal enzymatic reduction takes place [22], the external production of siderophores may provide an additional layer of defense. Additionally, as mentioned above, the siderophores' ability to reduce reactive oxygen species could help AAP as they need protection against oxidative stress due to their aerobic production of $\mathrm{BChl} a$.

Chromocurous halotolerans EG19, is a $\gamma$-proteobacterial AAP that was isolated during the spring of 2002 from floating microbial mats within the East German Creek System, Manitoba, Canada [23]. As this is a landlocked hypersaline spring system, it likely contains highly endemic communities of microorganisms that have not been mixed with or affected by allochthonous populations [23]. EG19 forms motile, short rod or longer curved rodshaped, orange-pink bacteria. When grown with complex carbon sources, EG19 produces a brown pigmented hydrophilic compound, which is excreted into the growth medium. While a similar phenomenon had never been reported in other AAP, it was hypothesized that the compound could be a siderophore [24], as ferric bound siderophores can be visually yellow-brown or red-brown [25]. Our study confirms the identity of this extracellular product and describes it as the first siderophore discovered in an AAP. Other AAP from a vast array of environments that do not pigment growth medium were also tested for their production of siderophores, as most of these metal chelating small molecules are colourless, and synthesis is therefore possible.

\section{Materials and Methods}

\subsection{Bacterial Strains and Growth Conditions}

For this study, 101 strains of AAP originating from an assortment of environments, as well as phylogenetically diverse throughout numerous proteobacterial clades were selected from Dr. Vladimir Yurkov's vast collection. A complete list of chosen strains, 
original source of isolation, relatedness to type species, and 16S rRNA partial gene sequence accession numbers are listed in Tables A1 and A2.

Freshwater AAP were cultivated on rich organic (RO) medium as described [26], with one minor modification. Bactopeptone was reduced to $0.5 \mathrm{~g} / \mathrm{L}$ and casamino acids were supplemented at $0.5 \mathrm{~g} / \mathrm{L}$, which provided a larger variety of complex nutrients. Marine AAP requiring salt were propagated on $\mathrm{RO}$ medium described above supplemented with $2 \% \mathrm{NaCl}$. AAP originally isolated from the East German Creek System, Manitoba, Canada, were grown using medium A (MA) [23]. Those isolated from biological soil crusts of Sandy Lands Forest and Spruce Woods National Park were cultured on Biological Soil Crust Medium A or B (BSCA or BSCB) [27]. Strains from meromictic Mahoney Lake in British Columbia, Canada were grown on N1 medium [28].

In addition to bacterial isolates formerly described elsewhere, some of those chosen for siderophore testing had not been previously published. AAP isolated from the meromictic Blue Lake in British Columbia, Canada, were cultured using a Blue Lake medium (BLM) containing (g/L): $\mathrm{MgSO}_{4}, 0.5 ; \mathrm{NH}_{4} \mathrm{Cl}, 0.3 ; \mathrm{KH}_{2} \mathrm{PO}_{4}, 0.3 ; \mathrm{KCl}, 0.3 ; \mathrm{CaCl}_{2}, 0.05 ; \mathrm{NaCl}, 12.0$; $\mathrm{NaHCO}_{3}, 0.5$; Na-acetate, 1.0; malic acid, 1.0; yeast extract, 1.0; bactopeptone, 0.5; with vitamins and trace elements solutions, $2.0 \mathrm{~mL}$ each; autoclaved at $\mathrm{pH} 5.9$ and then adjusted after autoclaving to $\mathrm{pH} 7.5$ with $0.5 \mathrm{~N} \mathrm{NaOH}$.

Furthermore, several AAP had been recovered from the freshwaters of Lake Winnipeg whilst the habitat was under study [29,30]. Specifically, in the spring of 2017, strain AJ72 was collected at Grand Beach from littoral water, AM19, AM27 and AM91 were from littoral sediment of Victoria Beach. During that summer, BA23 and BE100 were isolated at limnetic sites S1 and S5, respectively, while BL67 and BK61 originated within the littoral water and sediment of Grand Beach and Victoria Beach, respectively. Fall samples of Grand Beach sediment contained CK155 and CK182, while Victoria Beach littoral waters revealed isolate CL63. All were purified on the slightly modified freshwater RO medium as described.

\subsection{Iron Chelating Chromeazurol S Assay}

Every chosen strain was grown on $2 \%$ agar plates containing their specific growth medium supplemented with the dye chromeazurol S (CAS), which turns blue when bound to Fe and reverts to yellow/orange being released [31]. To make media, $60.5 \mathrm{mg}$ CAS were dissolved in $50 \mathrm{~mL}$ ddH $\mathrm{H}_{2} \mathrm{O}$, then mixed with $10 \mathrm{~mL}$ of an iron solution containing $1 \mathrm{mM}$

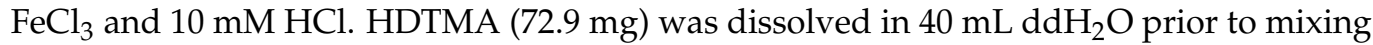
with the CAS/iron solution, bringing the total volume to $100 \mathrm{~mL}$. Separately, $900 \mathrm{~mL}$ of each growth medium (MA, $\mathrm{RO}, \mathrm{RO} 2 \% \mathrm{NaCl}, \mathrm{BSCA}$, or BLM) were prepared without the addition of iron, but with the correct amount of components for $1 \mathrm{~L}$, as that would be the final volume of medium after combining with CAS/iron solution. Media were then autoclaved at $\mathrm{pH} 5.9$, separate from the CAS mixture. After autoclaving, each medium was adjusted to $\mathrm{pH} 6.8$ and the CAS solution was added. Agar plates would turn blue when solidified. If measurements of ingredients were not exact or added in an incorrect order, CAS would precipitate out of solution and plates with $\mathrm{pH}$ higher than 6.8 would appear green instead of blue. CAS plates were heavily inoculated with each strain, and streaked to achieve isolated colonies after 5 days of growth at $28{ }^{\circ} \mathrm{C}$ in the dark.

\subsection{Variant Cation Chromeazurol S Assay}

Due to the principle chemistry behind the siderophore assay [31], which used negatively charged CAS dye that would weakly bind the cation $\mathrm{Fe}^{3+}$, we decided to replace $\mathrm{Fe}^{3+}$ with different metal(loid) cations, including $\mathrm{Mg}^{2+}, \mathrm{V}^{3+}, \mathrm{Mn}^{2+}, \mathrm{Co}^{2+}, \mathrm{Ni}^{2+}, \mathrm{Cu}^{2+}, \mathrm{Zn}^{2+}, \mathrm{Se}^{4+}$ and $\mathrm{Te}^{2+}$. Microbial growth with an aura of colour change in medium from blue to yellow hue would signify that microbially produced siderophore could bind the substituted cation, causing the released dye to revert to its yellow colour. These variant cation CAS plates were successfully created as explained in the previous section, cured to diverse shades of blue dependent on the metal(loid), and were screened for metallophore production identically 
as in the Fe-chelation CAS assay. All cations were purchased from Sigma Aldrich, USA as chloride salts, which were soluble once acidified as described.

\subsection{Phylogeny}

To assess the phylogenetic position, $16 \mathrm{~S}$ rRNA gene sequences were either acquired from previously published sources, or newly sequenced in this study. A list of accession numbers to partial 16S rRNA gene sequences attained from public repositories was provided in Tables A1 and A2. From unsequenced strains, DNA was extracted via the phenol chloroform method [32], and sequenced using Sanger technique [33]. Primer set 27F and 1492R, $5^{\prime}$-AGAGTTTGATCCTGGCTCAG-3' and 5'-GGTTACCTTGTTACGACTT-3', respectively, was used to achieve a total contiguous $16 \mathrm{~S}$ rRNA gene sequence length of $>1400$ bp per strain. New sequences of $16 \mathrm{~S}$ rRNA genes from previously unidentified AAP strains were deposited to GenBank under accession numbers (MW970346-MW970408) as listed in Tables A1 and A2. Genetic relation of $16 \mathrm{~S}$ rRNA gene sequences acquired from each AAP isolate were compared to the archived sequences of type species using the web-based software Basic Local Alignment Search Tool, BLAST [34]. Phylogenetic trees were constructed via MEGA X software [35] with 1000 bootstraps [36], using Maximum Likelihood method to align all AAP 16S rRNA gene sequences to one another based on the General Time Reversible model [37]. Initial tree(s) for the heuristic search were obtained automatically by applying Neighbor-Join and BioNJ algorithms to a matrix of pairwise distances estimated using the Maximum Composite Likelihood (MCL) approach, and then selecting the topology with superior log likelihood value.

\subsection{Siderophore Isolation and Concentration from C. halotolerans}

Strain EG19 was grown at $28{ }^{\circ} \mathrm{C}$ on MA agarized (2\%) plates in the dark for 5 days. Pink-purple colonies developed with brown hue dispersed in agarized medium (Figure 1A), which corresponded to zone of clearing on Fe-Chromeazurol S Assay (Figure 1B). To obtain liquid cultures, EG19 was inoculated at 5\% in Fe-free MA and grown for 5 days at $28{ }^{\circ} \mathrm{C}$ shaking at $200 \mathrm{rpm}$ in the dark. Fe-free MA was prepared as described in Chromeazurol $\mathrm{S}$ Assay, excluding Fe from the trace element solution. In addition, after all components were added, $5 \mathrm{~g} / \mathrm{L}$ of Chelex resin enclosed in dializing tubing was placed in the medium for $1 \mathrm{~h}$ prior to autoclaving. This step allowed the Chelex resin to remove traces of Fe introduced through the complex nutrients such as yeast extract, bactopeptone and casamino acids [38].

After the cultivation of $C$. halotolerans, Fe-free medium became dark brown. The pinkpurple cells were pelleted in $\sim 450 \mathrm{~mL}$ bottles at 10,000 rpm for $30 \mathrm{~min}$ using a Beckman J2HS centrifuge and a JA-10 rotor (Figure 1C). Siderophore containing supernatant was collected and 1.25 L was transferred into $2 \mathrm{~L}$ Erlenmeyer flasks (Figure 1D) to freeze overnight at $-20{ }^{\circ} \mathrm{C}$. Highly concentrated dark brown-pigmented high-salt slurry was formed predominantly on top of ice, while the lower part of ice was close to colourless. The flask with frozen material was removed from freezer and allowed to defrost at room temperature inserted upside down in a new collection beaker. Thawed supernatant was fractioned into $250 \mathrm{~mL}$ batches, with concentrated siderophore thawed and collected first, leaving frozen medium behind (Figure 1D), both observable visually (Figure 1E), and from the absorbance spectrum (Figure 1F).

A batch-type method of siderophore purification was used in this study [39]. Here, the combined freeze-concentrated samples were adjusted to $\mathrm{pH} 6.0$ and XAD 7-HP resin was added ( $20 \mathrm{~g}$ resin per L of supernatant). This slurry was shaken at $200 \mathrm{rpm}$ for $1 \mathrm{~h}$ on a rotary shaker and then filtered through a glass Millipore filter funnel that would collect resin, but allow supernatant to easily flow through. Concentrated siderophore required several extractions with resin to remove all pigment from supernatant. Siderophore-boundresin (now brown in colour) was thoroughly washed with $\mathrm{ddH}_{2} \mathrm{O}$ to remove all residual salts and other soluble contaminants. Vacuum assisted drying using Millipore system was performed, $\mathrm{ddH}_{2} \mathrm{O}$ discarded, and the cleaned siderophore-bound-resin was soaked 
in methanol for $30 \mathrm{~min}$ to release the siderophore. Pigmented methanol was collected, and resin was soaked once more using the same batch of solvent for additional extraction. Methanol extracts were combined and then evaporated to dryness. The resulting dark brown powder was the presumed highly concentrated siderophore, and kept at $-20{ }^{\circ} \mathrm{C}$ until further testing. Unbound-resin was washed with methanol, then soaked with $\mathrm{ddH}_{2} \mathrm{O}$ prior to reuse.

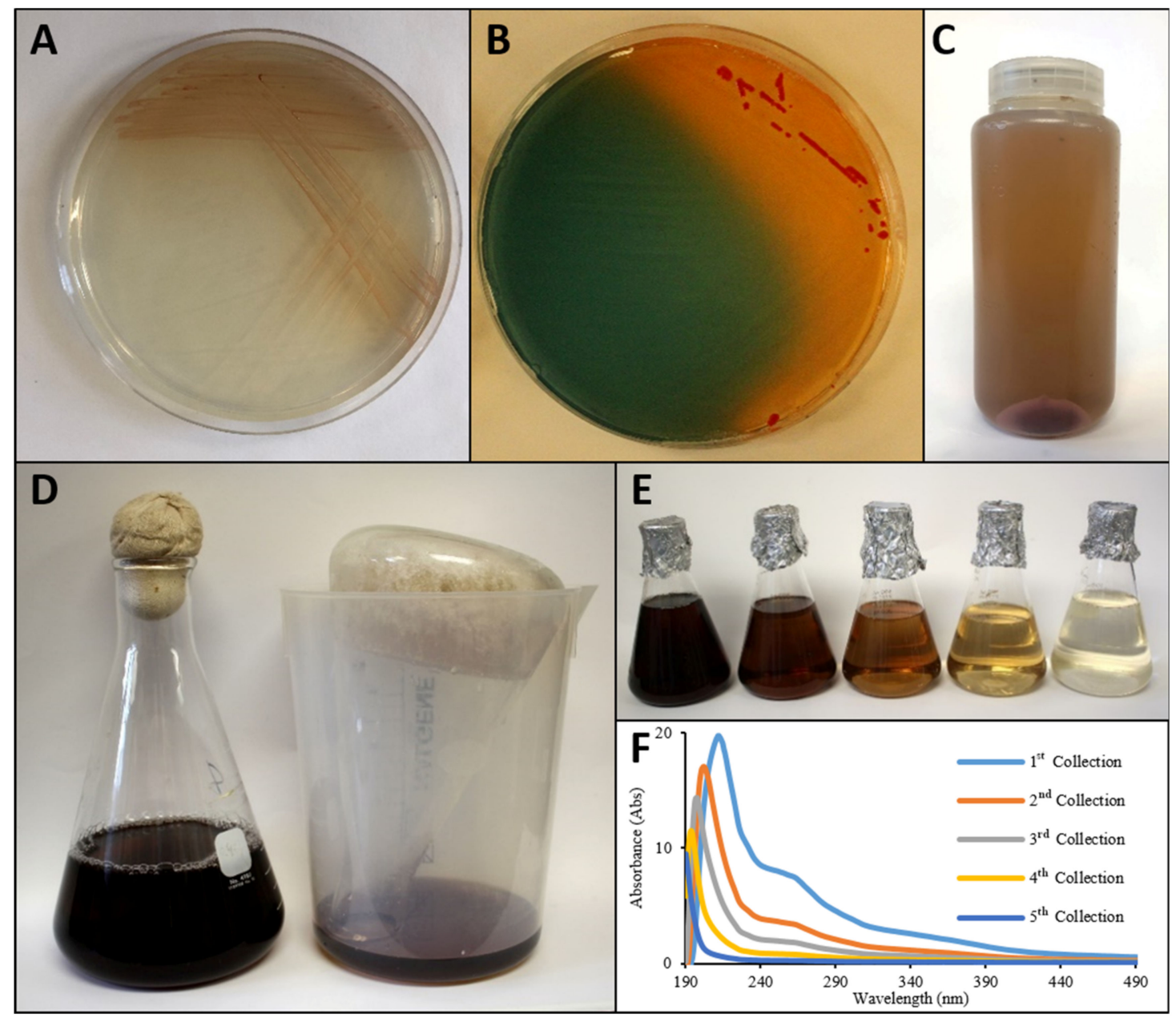

Figure 1. Extraction and concentration of brown pigment excreted by C. halotolerans. (A) Strain EG19 released a brown pigment into MA medium. (B) Fe-CAS plate with siderophore production by EG19. (C) Pelleted cells under supernatant. (D) Pigment concentrated with freezing-out technique. (E) Collected fractions during thawing with concentrated pigment released first. (F) Absorbance spectrum of each fraction.

\subsection{Purification and $\mathrm{Fe}$-Chelation of Siderophore from $\mathrm{C}$. halotolerans}

Concentrated siderophore powder from resin purification was resuspended in $\mathrm{ddH}_{2} \mathrm{O}$ (10 $\mathrm{mg}$ in $50 \mu \mathrm{L}$ ), and decimally diluted up to $10^{-3}$. In addition, a solution containing $5 \mathrm{mg}$ of concentrate in $100 \mu \mathrm{L}$ ddH ${ }_{2} \mathrm{O}$ was filtered through an Amicon ${ }^{\circledR}$ Ultra $0.5 \mathrm{~mL} 3000$ Da spin column manufactured by Millipore Ireland, which removed any contaminating proteins over that size. This filtered concentrate was also decimally diluted up to $10^{-2}$. Once the dilutions were made, $10 \mu \mathrm{L}$ of each sample, and an aliquot of freeze-thawed concentrate, were individually mixed with $10 \mu \mathrm{L}$ of loading buffer, prior to filling into the wells of a Mini-Protean tris-tricine gel, 16.5\% from Bio-Rad, Hercules, CA, USA. Loading buffer contained $200 \mathrm{mM}$ tris- $\mathrm{HCl}, \mathrm{pH}$ 6.8, 2\% SDS, 40\% glycerol and 0.04\% Coomassie Brilliant Blue G-250 (CBB) from Bio-Rad, USA. Running buffer was made up of $1 \mathrm{M}$ tris, $1 \mathrm{M}$ tricine and $1 \%$ SDS at $\mathrm{pH} 8.3$. Wells were $30 \mu \mathrm{L}$, filled with $20 \mu \mathrm{L}$ of sample $(10 \mu \mathrm{L}$ of sample, $10 \mu \mathrm{L}$ of loading buffer). Protein ladder was a C6210 - color marker ultra-low range (M.W. 
1060-26,600) from Sigma-Aldritch, St. Louis, MO, USA. Gel electrophoresis was run at 100 volts for $1 \mathrm{~h}$ prior to being stained in a CBB solution $(1 \mathrm{~g}$ of CBB dissolved in $1 \mathrm{~L}$ of [50\% MeOH, $10 \%$ glacial acetic acid, $\left.40 \% \mathrm{H}_{2} \mathrm{O}\right]$ ]). The gel was then destained with a mixture containing $5 \%$ acetic acid and $20 \% \mathrm{MeOH}$ overnight.

Fe chelation was tested with the concentrated siderophore powder from the resin concentration step, the brown pigment filter-purified below $3 \mathrm{kDa}$, as well as with a sample from the remaining proteins that were $>3 \mathrm{kDa}$ from the spin filtration procedure. Each fraction was solubilized, where $10 \mathrm{mg}$ of dried pigment was dissolved in $500 \mu \mathrm{L}$ of $60 \%$ $\mathrm{MeOH}$, prior to applying each solution into a blank diffusion disk, manufactured by Oxoid in the UK, allowing it to become dry and concentrated within the disk. These were then placed on a Fe-CAS plate and left to react with chromeazurol S overnight.

\section{Results}

\subsection{Bacterial Growth and Fe-CAS-Plate Reactions}

Comparative phenotypic analysis was achieved by allowing each strain to develop for 5 days on their respective agarized media under conditions that promoted the best growth. This elapsed time ensured stationary phase was reached for each representative, which resulted in the formation of sufficient colonies for analysis. Triplicate CAS-supplemented and CAS-free controls were simultaneously plated to identify the viability of inoculum. In all cases, growth occurred on CAS plates, but was marginally reduced in comparison to controls. Both after 3 and 5 days, the average zone of colour change (blue to yellow) was recorded, revealing several phenotypic attributes. As some strains grew slower than others, 5 days' period was chosen to analyze and compare all simultaneously. We identified siderophore production based on zone of diffusion/colour change around colonies as follows (Figure A1): no zone $(-)$, a zone $<1 \mathrm{~mm}$ around colonies $(+)$, a moderate diffusion $<10 \mathrm{~mm}(++)$, and considerable diffusion $>10 \mathrm{~mm}(+++)$. Fe-chelating CAS reactions after 5 days are listed in Tables 1 and 2, as well as shown in Figure 2 beside each strain name.

\subsection{Substitute Cation CAS Assays}

Once Fe-chelating siderophore production was confirmed using the standard Fe-CAS assay, all strains were tested on CAS supplemented agar plates that contained one of 9 other metal(loid) cations: $\mathrm{Mg}^{2+}, \mathrm{V}^{3+}, \mathrm{Mn}^{2+}, \mathrm{Co}^{2+}, \mathrm{Ni}^{2+}, \mathrm{Cu}^{2+}, \mathrm{Zn}^{2+}, \mathrm{Se}^{4+}$ and $\mathrm{Te}^{2+}$. Results for varied CAS assays are listed along-side $\mathrm{Fe}^{2+}$ data in Tables 1 and 2 using the same zone distinctions as described. While some strains only produced siderophores that reacted with $\mathrm{Fe}^{2+}$, others had secondary metabolites capable of chelating additional metal(loid) cations. Most strains capable of chelating metals other than Fe could also chelate iron, with the exception of two strains P4 and SS56 (Figure A2), which were found to produce metallophore capable of acting predominantly on other tested metal(loid)s, rather than Fe.

\subsection{Phylogenetic Diversity of Siderophore Producing AAP}

Isolates were chosen to represent AAP from a variety of environments as well as embody a host of phylogenetically diverse species. In this way, the 101 representatives listed in Tables 1 and 2 were cultivated, and 16S rRNA gene sequences acquired either from repositories, or decoded in this work. Phylogenetic relation to sequences of known type strains was determined by BLAST search (Tables A1 and A2). In addition, these sequences were used to create a phylogenetic tree (Figure 2), that also included some previously described type species not tested for siderophore production, but were included as key placeholders of phylogenetic groups. The evolutionary analysis performed on Mega X using Maximum Likelihood method involved 132 nucleotide sequences and had a total of 1717 positions in the final data set. The chosen AAP diversely spread throughout Erythrobacteraceae and Sphingomonadaceae relating to known AAP type species. While some aligned to reported AAP in Acetobacteraceae, and Rhodobacteraceae, many others aligned to organisms previously undescribed as AAP within these clades, as well as to some species within Hyphomonadaceae and Methylobacteraceae. 


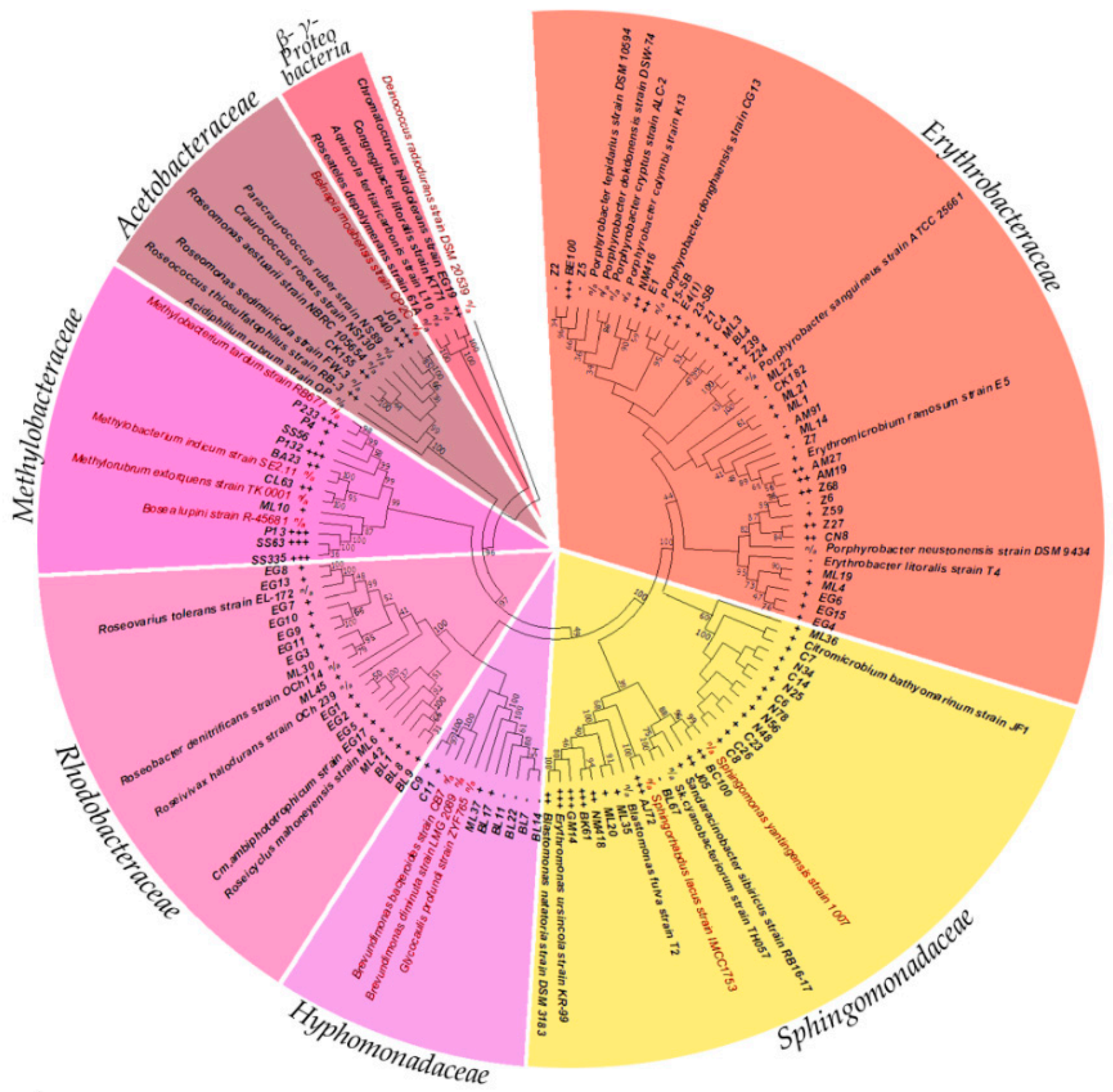

Figure 2. Phylogenetic tree of AAP tested for siderophore production. Isolates hailed from $\alpha$-proteobacterial families Erythrobacteraceae, Sphingomonadaceae, Acetobacteraceae, Rhodobacteraceae, Hyphomonadaceae and Methylobacteraceae, as well as a representative within the $\gamma$ - subclass of Proteobacteria (titled sections around circumference of circle). Fe-chelation siderophore activities are listed between strain names and phylogenetic position. Strain names in bold are confirmed AAP, red highlighted are not. 
Table 1. Freshwater and saline AAP analyzed for metal(loid)-chelation via CAS assays with varied cations.

\begin{tabular}{|c|c|c|c|c|c|c|c|c|c|c|c|c|}
\hline Environment & Strain & Medium & Mg & $\mathbf{V}$ & Mn & $\mathrm{Fe}$ & Co & $\mathrm{Ni}$ & $\mathrm{Cu}$ & $\mathrm{Zn}$ & Se & $\mathrm{Te}$ \\
\hline $\begin{array}{l}\text { Hot spring, Kamchatka } \\
\text { Isl. }\end{array}$ & $\mathrm{KR} 9^{\mathrm{T}}$ & $\mathrm{RO}$ & ++ & +++ & ++ & +++ & ++ & ++ & ++ & +++ & ++ & +++ \\
\hline \multirow{6}{*}{$\begin{array}{l}\text { Warm temperature } \\
\text { spring, Baikal Lake, } \\
\text { Russia }\end{array}$} & E1 & $\mathrm{RO}$ & - & - & + & ++ & + & + & - & - & - & - \\
\hline & $\mathrm{E} 4(1)$ & $\mathrm{RO}$ & - & - & - & ++ & - & - & - & - & - & + \\
\hline & $\mathrm{E} 5^{\mathrm{T}}$ & $\mathrm{RO}$ & - & - & - & + & - & - & - & - & - & + \\
\hline & $\mathrm{RB} 3^{\mathrm{T}}$ & $\mathrm{RO}$ & - & - & ++ & ++ & - & ++ & + & + & + & + \\
\hline & RB16-17 ${ }^{\mathrm{T}}$ & $\mathrm{RO}$ & - & - & - & + & - & - & + & - & - & - \\
\hline & $\mathrm{T}^{\mathrm{T}}$ & $\mathrm{RO}-\mathrm{NaCl}$ & + & + & + & - & + & + & - & - & + & + \\
\hline \multirow{12}{*}{$\begin{array}{c}\text { Deep Ocean, Juan De } \\
\text { Fuca Ridge, Pacific } \\
\text { Ocean }\end{array}$} & $\mathrm{JF} 1^{\mathrm{T}}$ & $\mathrm{RO}-\mathrm{NaCl}$ & + & + & + & + & + & + & + & + & + & + \\
\hline & $\mathrm{C} 6$ & $\mathrm{RO}-\mathrm{NaCl}$ & + & + & + & + & + & + & + & + & + & + \\
\hline & $\mathrm{C} 7$ & $\mathrm{RO}-\mathrm{NaCl}$ & + & + & + & + & - & + & + & + & + & + \\
\hline & $\mathrm{C} 8$ & $\mathrm{RO}-\mathrm{NaCl}$ & + & + & + & + & - & + & + & + & + & + \\
\hline & C14 & $\mathrm{RO}-\mathrm{NaCl}$ & + & + & + & + & - & + & + & + & + & + \\
\hline & $\mathrm{C} 23$ & $\mathrm{RO}-\mathrm{NaCl}$ & - & + & + & + & + & + & + & + & + & + \\
\hline & C26 & $\mathrm{RO}-\mathrm{NaCl}$ & + & + & + & + & + & + & + & + & + & + \\
\hline & N25 & $\mathrm{RO}-\mathrm{NaCl}$ & + & + & + & + & + & + & + & + & + & + \\
\hline & N34 & $\mathrm{RO}-\mathrm{NaCl}$ & + & + & + & + & + & + & + & + & + & + \\
\hline & N48 & $\mathrm{RO}-\mathrm{NaCl}$ & + & + & + & + & + & + & + & + & + & + \\
\hline & N56 & $\mathrm{RO}-\mathrm{NaCl}$ & + & + & + & + & + & + & + & + & + & + \\
\hline & N78 & $\mathrm{RO}-\mathrm{NaCl}$ & + & + & + & + & + & + & + & + & + & + \\
\hline \multirow{2}{*}{$\begin{array}{l}\text { Rag Beach sediment, } \\
\text { BC, Canada }\end{array}$} & $15-S B$ & $\mathrm{RO}-\mathrm{NaCl}$ & + & - & + & + & - & - & + & + & + & + \\
\hline & 23-SB & $\mathrm{RO}-\mathrm{NaCl}$ & + & - & + & + & + & + & - & + & - & - \\
\hline \multirow{5}{*}{$\begin{array}{c}\text { Central Gold Mine, MB, } \\
\text { Canada }\end{array}$} & C4 & $\mathrm{RO}$ & - & + & + & + & + & + & + & + & - & + \\
\hline & C9 & $\mathrm{RO}$ & - & + & - & + & + & - & + & + & - & + \\
\hline & C11 & $\mathrm{RO}$ & - & + & - & + & - & - & + & + & - & - \\
\hline & NM4.16 & $\mathrm{RO}$ & - & + & + & ++ & + & + & + & + & - & + \\
\hline & NM4.18 & $\mathrm{RO}$ & ++ & + & + & ++ & ++ & ++ & ++ & + & ++ & ++ \\
\hline \multirow{14}{*}{$\begin{array}{c}\text { Lake Winnipeg, MB, } \\
\text { Canada }\end{array}$} & AJ 72 & $\mathrm{RO}$ & - & + & + & +++ & + & + & + & ++ & - & + \\
\hline & AM 19 & $\mathrm{RO}$ & - & + & - & +++ & - & - & - & ++ & - & - \\
\hline & AM 27 & $\mathrm{RO}$ & - & - & - & ++ & - & - & - & + & - & - \\
\hline & AM 91 & $\mathrm{RO}$ & - & - & - & - & - & - & - & - & - & - \\
\hline & BA 23 & $\mathrm{RO}$ & + & ++ & ++ & ++ & ++ & + & ++ & ++ & ++ & ++ \\
\hline & BC 100 & $\mathrm{RO}$ & - & - & - & ++ & + & - & - & - & - & - \\
\hline & BE 100 & $\mathrm{RO}$ & ++ & ++ & ++ & +++ & ++ & ++ & ++ & ++ & ++ & + \\
\hline & BK 61 & $\mathrm{RO}$ & + & + & + & +++ & + & - & + & ++ & + & - \\
\hline & BL 67 & $\mathrm{RO}$ & - & - & - & - & + & - & - & - & - & - \\
\hline & CK 155 & $\mathrm{RO}$ & - & - & - & ++ & - & - & - & - & - & - \\
\hline & CK 182 & $\mathrm{RO}$ & - & - & - & - & - & - & - & - & - & - \\
\hline & CL 63 & $\mathrm{RO}$ & + & ++ & ++ & ++ & ++ & ++ & ++ & ++ & ++ & ++ \\
\hline & CN8 & $\mathrm{RO}$ & + & ++ & + & ++ & + & + & - & ++ & ++ & + \\
\hline & GM14 & $\mathrm{RO}$ & ++ & ++ & ++ & +++ & ++ & + & - & ++ & ++ & + \\
\hline \multirow{10}{*}{$\begin{array}{c}\text { Zebra mussels, Lake } \\
\text { Winnipeg, MB, Canada }\end{array}$} & $\mathrm{Z1}$ & $\mathrm{RO}$ & - & - & - & + & - & - & - & - & - & - \\
\hline & $\mathrm{Z} 2$ & $\mathrm{RO}$ & - & - & - & - & - & - & - & - & - & - \\
\hline & Z5 & $\mathrm{RO}$ & - & - & - & - & - & - & - & - & - & - \\
\hline & Z6 & $\mathrm{RO}$ & + & - & + & - & + & + & - & - & + & - \\
\hline & $\mathrm{Z7}$ & $\mathrm{RO}$ & + & - & - & - & - & - & - & - & - & - \\
\hline & Z24 & $\mathrm{RO}$ & - & - & - & ++ & - & - & - & + & - & - \\
\hline & Z27 & $\mathrm{RO}$ & + & + & + & ++ & - & - & - & ++ & + & ++ \\
\hline & Z39 & $\mathrm{RO}$ & - & - & + & ++ & + & - & - & + & - & - \\
\hline & Z59 & $\mathrm{RO}$ & + & - & + & + & - & + & - & - & + & - \\
\hline & Z68 & $\mathrm{RO}$ & - & ++ & - & ++ & - & + & - & ++ & - & + \\
\hline
\end{tabular}


Table 2. Meromictic lake, saline spring and biological soil crust AAP analyzed for metal(oid)-chelation via CAS assays with varied cations.

\begin{tabular}{|c|c|c|c|c|c|c|c|c|c|c|c|c|}
\hline Environment & Strain & Medium & $\mathrm{Mg}$ & $\mathbf{V}$ & Mn & $\mathrm{Fe}$ & Co & $\mathbf{N i}$ & $\mathrm{Cu}$ & $\mathrm{Zn}$ & Se & $\mathrm{Te}$ \\
\hline \multirow{16}{*}{$\begin{array}{c}\text { Mahoney Lake, BC, } \\
\text { Canada }\end{array}$} & ML1 & N1 & - & + & - & + & - & - & + & + & - & + \\
\hline & ML3 & N1 & - & + & - & + & - & - & + & + & - & + \\
\hline & $\mathrm{ML}_{4}^{\mathrm{T}}$ & N1 & - & + & - & + & - & - & + & + & - & + \\
\hline & ML6 $^{\mathrm{T}}$ & N1 & + & + & + & + & - & - & + & + & - & + \\
\hline & ML10 & N1 & - & + & - & + & - & - & + & + & - & + \\
\hline & ML14 & N1 & - & + & - & + & - & - & + & + & - & + \\
\hline & ML19 & N1 & + & + & + & + & + & - & + & + & + & + \\
\hline & ML20 & N1 & - & + & - & + & - & - & + & + & - & + \\
\hline & ML21 & N1 & - & + & - & + & - & - & + & + & - & + \\
\hline & ML22 & N1 & - & + & + & + & - & - & + & + & - & + \\
\hline & ML30 & N1 & + & + & - & + & - & - & + & + & + & + \\
\hline & ML35 & N1 & - & + & - & + & - & - & + & + & - & + \\
\hline & ML36 & N1 & + & + & + & + & + & - & + & + & + & + \\
\hline & ML37 & N1 & - & + & + & + & - & - & + & + & - & + \\
\hline & ML42 & N1 & + & + & + & + & + & + & + & + & + & + \\
\hline & ML45 & N1 & + & + & + & + & - & + & + & + & + & + \\
\hline \multirow{10}{*}{ Blue Lake, BC, Canada } & BL1 & BLM & + & + & + & + & + & + & + & + & + & + \\
\hline & BL4 & BLM & - & + & - & + & - & - & + & + & - & + \\
\hline & BL5 & BLM & + & + & + & + & + & + & + & + & + & + \\
\hline & BL7 & BLM & + & + & + & - & + & + & + & - & + & + \\
\hline & BL8 & BLM & + & + & + & + & + & + & + & + & + & + \\
\hline & BL9 & BLM & + & + & + & + & + & + & + & + & + & + \\
\hline & BL11 & BLM & + & + & + & - & + & + & + & + & + & + \\
\hline & BL14 & BLM & + & + & + & - & + & + & + & + & + & + \\
\hline & BL17 & BLM & + & + & + & + & + & + & + & + & + & + \\
\hline & BL22 & BLM & + & - & + & - & + & + & + & - & + & + \\
\hline \multirow{15}{*}{$\begin{array}{l}\text { East German Creek } \\
\text { System, MB, Canada }\end{array}$} & EG1 & MA & - & + & - & + & - & - & + & + & - & + \\
\hline & EG2 & MA & + & + & + & + & - & - & + & + & - & + \\
\hline & EG3 & MA & - & - & - & + & - & - & - & - & - & - \\
\hline & EG4 & MA & - & + & - & + & - & - & + & + & - & + \\
\hline & EG5 & MA & - & + & + & + & - & - & + & + & - & + \\
\hline & EG6 & MA & - & + & - & + & - & - & + & + & - & + \\
\hline & EG7 & MA & - & + & + & + & - & - & + & + & - & + \\
\hline & EG8 & MA & - & + & - & + & + & - & + & + & + & + \\
\hline & EG9 & MA & - & + & - & + & - & - & + & + & - & + \\
\hline & EG10 & MA & - & + & - & + & - & - & + & + & + & + \\
\hline & EG11 & MA & - & + & - & + & - & - & + & + & - & + \\
\hline & EG13 & MA & - & + & - & + & - & - & + & + & - & + \\
\hline & EG15 & MA & - & + & - & + & + & - & + & + & - & + \\
\hline & $\mathrm{EG} 17^{\mathrm{T}}$ & MA & + & + & + & + & + & + & + & + & - & + \\
\hline & EG19 ${ }^{\mathrm{T}}$ & MA & + & + & + & ++ & - & + & + & ++ & + & + \\
\hline \multirow{3}{*}{$\begin{array}{c}\text { Sandy Lands Forest soil } \\
\text { crust, MB, Canada }\end{array}$} & SS56 & BSCA & ++ & ++ & ++ & + & ++ & ++ & ++ & ++ & ++ & ++ \\
\hline & SS63 & BSCA & ++ & +++ & ++ & +++ & ++ & +++ & +++ & +++ & +++ & +++ \\
\hline & SS335 & BSCA & +++ & +++ & +++ & +++ & ++ & ++ & +++ & ++ & ++ & +++ \\
\hline \multirow{7}{*}{$\begin{array}{c}\text { Spruce Woods National } \\
\text { Park soil crust, MB, } \\
\text { Canada }\end{array}$} & J01 & BSCA & +++ & ++ & +++ & +++ & + & ++ & ++ & ++ & + & ++ \\
\hline & J05 & BSCA & + & + & + & ++ & - & - & + & + & - & + \\
\hline & $\mathrm{P} 4$ & BSCB & ++ & ++ & ++ & + & + & + & ++ & ++ & ++ & ++ \\
\hline & P13 & BSCB & +++ & +++ & +++ & +++ & ++ & +++ & +++ & +++ & ++ & ++ \\
\hline & P40 & BSCB & ++ & ++ & ++ & ++ & + & ++ & ++ & ++ & + & + \\
\hline & P132 & BSCB & ++ & ++ & ++ & +++ & ++ & ++ & ++ & ++ & ++ & ++ \\
\hline & P233 & BSCB & - & ++ & ++ & +++ & ++ & ++ & ++ & ++ & ++ & ++ \\
\hline
\end{tabular}




\subsection{C. halotolerans Pigment Purification and Identification}

When purified via $16.5 \%$ tris-tricine gel electrophoresis, the brown pigment migrated further than the loading buffer's running dye, CBB, after $1 \mathrm{~h}$ (Figure 3A). This gel-shift revealed the pigment under study to be smaller than CBB, which has a known molecular weight of $856.03 \mathrm{~g} / \mathrm{mol}$. Since well 1 contained a standard ladder, the measurement of migration distance for the siderophores' brown band, and of each protein in the ladder allowed for the rough estimation of pigment size to be near 341 Da (Figure S1). Gel staining and destaining revealed that the siderophore sample collected after resin concentration and diluted in methanol at 200,20,2, and $0.2 \mu \mathrm{g} / \mathrm{mL}$ loaded into wells 2 through 5, respectively, had some contaminating small proteins as expected (Figure 3B). In addition, samples that received the subsequent removal of proteins larger than $3 \mathrm{kDa}$ via spin-column were also run on the same gel. Wells 6 through 8 contained brown pigment, which passed through the $<3 \mathrm{kDa}$ spin column and then diluted in $60 \%$ methanol to 100,10 , and $1 \mu \mathrm{g} / \mathrm{mL}$, respectively. This step purified the brown pigment of any contaminating proteins (Figure 3A,B). Often, small molecules below $1 \mathrm{kDa}$ are lost from the gel during destaining step [40], which likely caused the small brown pigment to escape similarly to CBB, (Figure 3B). Regardless, testing the crude dried pigment (Disk 1), as well as fractions $<3 \mathrm{kDa}$ and $>3 \mathrm{kDa}$ (Disks 2 and 3), confirmed the smaller fraction containing brown pigment acted as a siderophore, while the larger proteins did not (Figure $3 \mathrm{C}$ ). Since the $<3 \mathrm{kDa}$ fraction had no contaminants (Figure 3B), but contained the brown pigment prior to destaining (Figure 3A), the small $\sim 341$ Da molecule produced by C. halotolerans acted as a siderophore.

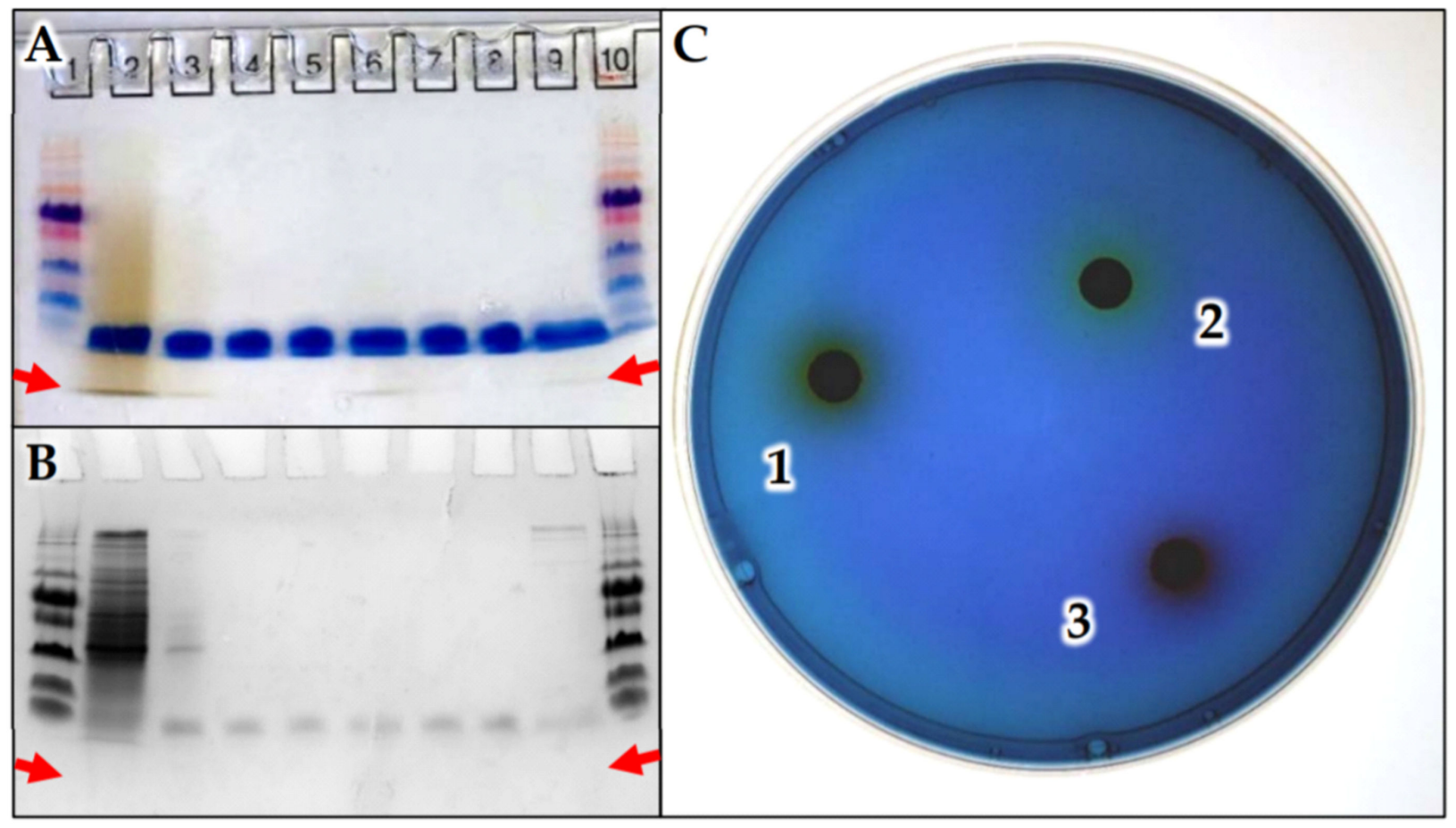

Figure 3. Gel purification of C. halotolerans brown pigment, and confirmation of siderophore activity. (A) Unstained and (B) stained tris-tricine gel electrophoresis performed on: siderophore from resin concentration, wells 2-5; siderophore sample smaller than $3 \mathrm{kDa}$, wells 6-8; proteins larger than $3 \mathrm{kDa}$ and remaining in solution well 9; standard ladder, wells 1 and 10. (C) Siderophore activity observed for: (1) Crude resin extract positive reaction visible as yellowing area; (2) $<3 \mathrm{kDa}$ dark brown fraction produced positive yellowing reaction; (3) Proteins $>3 \mathrm{kDa}$ negative results observed as darkening of blue due to alkaline $\mathrm{pH}$, without siderophore activity present. 


\section{Discussion}

\subsection{Siderophore Production Revealed by CAS-Assay}

While effectively employed for the identification of numerous siderophore producing bacteria, the CAS assay has a notable limitation; microbial growth may be hindered due to a few factors [31]. Since the metal cation of interest weakly bound to the dye, it was less freely diffusible into the bacteria, resulting in lower availability. In addition, the medium needed to be at $\mathrm{pH} 6.8$ for the indicative colour change to occur successfully. Finally, HDTMA has been known for its slight bacterial toxicity. If an organism had weak Fe transport, required a basic or acidic optimal $\mathrm{pH}$, or was sensitive to HDTMA, it would of had reduced growth. In our experiments, we found that while a range of media compositions could be used, all AAP did have reduced growth on CAS plates, when compared to control. However, since growth did indeed occur, siderophore production could therefore be analyzed.

Other, more high-throughput, alternative methods were considered, but the chosen agarized CAS-assay was most ideal for determining siderophore and metallophore production. Recently, a bulk screening assay for siderophore detection was proposed [41]. However, both the "traditional" and "modified microplate" qualitative techniques described could not be used for our application, since there was an assumption that siderophores were always constitutively expressed, whereas cultures were grown in complex media without manipulating the concentration of any metal of interest. While this may be the case for some strains, the expression/production of most siderophores or other secondary metabolites usually requires induction from an external factor, which can be either the presence or absence of a stimuli [18]. For biologically significant metals, including Fe, $\mathrm{Zn}$, and $\mathrm{Cu}$, the related cation-specific metallophores are expected to be produced only under limiting conditions. In opposite, metallophores that act on $\mathrm{V}, \mathrm{Te}, \mathrm{Se}$, or other more toxic metal(loid)s are presumably only synthesized when such toxins are present at higher concentrations. Therefore, growth on agarized plates that contained each metal of interest pre-bound to CAS-reagent as a stimulant for metallophore production was our chosen method. Future works are required to test varying concentrations of metal cations to determine which yields more/less production of specific metallophores.

Regarding the Fe-chelation, a range of phenotypes was observed, when detecting siderophore activity after the 5 -day incubation (Figure A1). In particular, 4 phenotypes were distinguished among all isolates based on size of clearing/colour change zone. Negative results $(-)$ had to have bacterial growth, but without a change in medium opacity. The smallest zone of clearing $(+)$, seen previously after prolonged growth [42], was likely not due to siderophore production [43]. Rather, this small aura could be due to high rates of metal uptake from the surrounding medium. Prolonged bacterial metabolism allowed for increased simple metal diffusion into cells, which reduced its amount in the near-by medium, rendering the dye in that narrow area void of metal, turning yellow. Hence, there is a very small $<1 \mathrm{~mm}$ zone. In comparison, moderate or highly diffusible siderophore release was quite evident, and was segregated into two phenotypic groups. A zone $<10 \mathrm{~mm}$ compared to a zone $>10 \mathrm{~mm}$, where each represented less or more diffusible secondary metabolite, respectfully, and where lower or higher concentrations were produced and captured additional metal cations. In this test, all 4 zonal varieties for Fe-chelation were discovered (Tables 1 and 2, and Figure 2).

Modifying the CAS assay to monitor chelation of elements other than $\mathrm{Fe}^{2+}$ included $\mathrm{Mg}^{2+}, \mathrm{V}^{3+}, \mathrm{Mn}^{2+}, \mathrm{Co}^{2+}, \mathrm{Ni}^{2+}, \mathrm{Cu}^{2+}, \mathrm{Zn}^{2+}, \mathrm{Se}^{4+}$ and $\mathrm{Te}^{2+}$ (Figure 4A). The method could be successfully adapted for all selected metal(loid)s, where only $\mathrm{Mn}^{2+}, \mathrm{Co}^{2+}, \mathrm{Ni}^{2+}, \mathrm{Cu}^{2+}$, $\mathrm{Zn}^{2+}$ had been proposed previously [44]. In addition, the assay could be used with variable nutrient and organic carbon concentrations that did not inhibit the activity. Furthermore, we discovered that a wide range of AAP produced metallophores, which bound a variety of metal(loid)s in addition to Fe (Tables 1 and 2). Some AAP had siderophores that specifically bound Fe only, including strains E1, E4(1), RB3, NM416, AM27, CK155, BC100, Z24, Z39, and J05. Most AAP with highly diffusible siderophores, and $>10 \mathrm{~mm}$ zones on Fe-CAS plates, had activity towards a large variety of metal(loid)s. Few strains, including SS56 and 
P4 preferentially bound metal(loid)s other than Fe. Since all 9 additional cations $\mathrm{Mg}^{2+}, \mathrm{V}^{3+}$, $\mathrm{Mn}^{2+}, \mathrm{Co}^{2+}, \mathrm{Ni}^{2+}, \mathrm{Cu}^{2+}, \mathrm{Zn}^{2+}, \mathrm{Se}^{4+}$ and $\mathrm{Te}^{2+}$ could be bound by metallophores, future work may consider a broader range of cations and the extent of metals that can be exogenously chelated. To determine if specific metal(loid)s were more readily chelated than others, all positive CAS assay results were tallied for each, Figure 4B. Here, multiple +++ represented strong production or significant interaction, and could be compared to weaker reactions such as + , or ++ . In this way, Fe was the highest cumulative acquired cation, with the activity ranked as $\mathrm{Fe}>\mathrm{Zn}>\mathrm{V}>\mathrm{Te}>\mathrm{Cu}>\mathrm{Mn}>\mathrm{Mg}>\mathrm{Se}>\mathrm{Ni}>\mathrm{Co}$. It would appear slight variation in cation size, from Fe (55.85) to Co (58.93), had a strong impact on activity, where Fe was most frequently captured, and Co the rarest. Indeed, since strongest reactions existed for Fe and $\mathrm{Zn}$, with less reactivity found for metals in between the sizes of these two within the periodic table, specific mechanisms likely existed to capture either metal, inferring cation specificity for each metallophore. Further analysis will be required to see if the broad range metal(loid) acquisition is due to the production of a single siderophore that reacts with a variety of metals, or the bacteria produced specific metallophores for each. AAP metallophore production could be explained by requirement of trace elements and the need of toxicity prevention. Both Fe and $\mathrm{Zn}$ are biologically necessary and commonly limited or unavailable in dissolved forms, therefore acquisition via specific siderophores would be an asset. The 3rd and 4th highest captured metal(loid)s were $\mathrm{V}$ and Te, which have known toxic properties, and could have been sequestered as a result of a protection mechanism alone. Bound to a metallophore, these cations would be too large to freely diffuse through outer membranes and be restricted from entering the cell, to prevent any toxic influence. The remaining 6 had reduced activity, likely because they are less toxic and easier available in microbial environment, and therefore less metallophores could be expected.

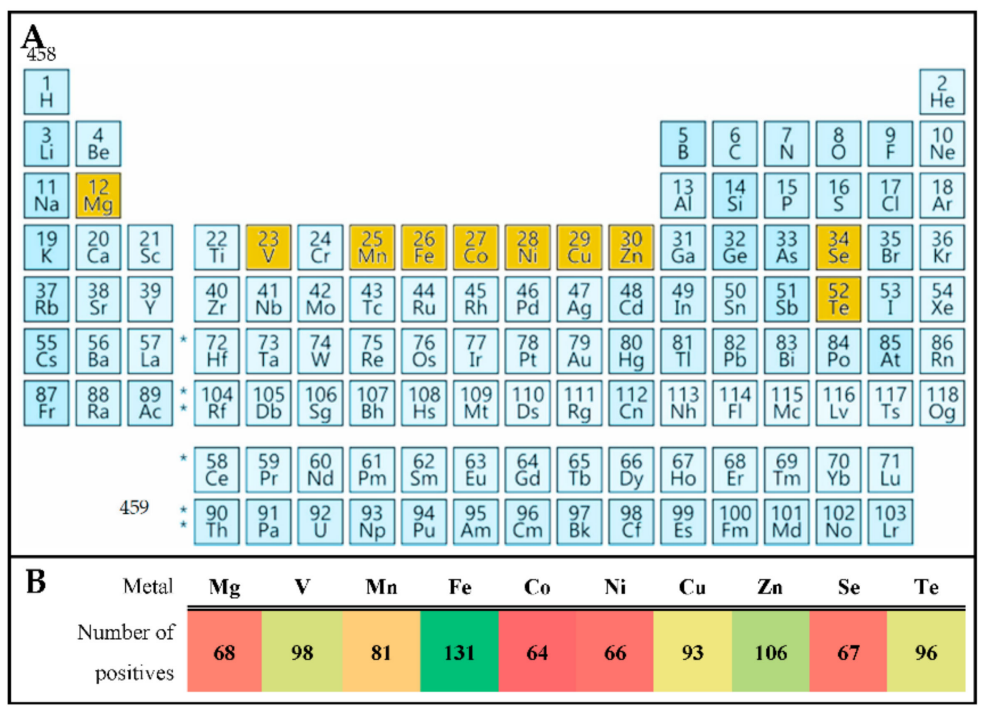

Figure 4. Variant metal(loid)s tested in CAS assay. (A) Chosen elements highlighted in yellow. (B) Tallied number of positive results from Tables 1 and 2.

\subsection{Environmental Distribution of Siderophore Producers}

In relation to the origin of isolation a few patterns were observed. Collections of AAP strains that originated from hot springs, freshwater lakes, and biological soil crusts all had a high proportion of siderophore producers. In opposition, the isolates from marine, meromictic lake, and saline spring habitats produced less, or no siderophores. Rather, they seem to rely on sufficient metal uptake directly from the local microenvironment by diffusion. The main differentiating factor in this case is the requirement of $\mathrm{NaCl}$. It appears that AAP capable of halotolerance or halophilic growth do not produce siderophores of equal activity or quantity as bacteria that do not depend on $\mathrm{NaCl}$ for growth. This 
correlation may be due to each strains' reaction to osmotic pressure. Cells that are adapted to tolerate higher levels of $\mathrm{NaCl}$ will likely have additional cation pumps to survive naturally and resist the high levels of solutes in a saline environment. As seen elsewhere, cation pumps can be non-specific, where many are capable of removing several toxic cations from the cytoplasm of microorganisms [45]. In comparison, freshwater AAP are comparably less osmophilic, and would therefore have less need of copious cation pumps in membranes. Therefore, they are more likely to evolve defensive mechanisms that modify the local environment to suit their needs, including the production of external small molecules that would render cations less diffusible.

\subsection{Phylogenetic Diversity of Fe-Chelating AAP}

Comparing phylogenetic diversity and the production of siderophores on CAS plates by AAP delivered a few notable trends (Figure 2). Broadly, no studied representatives of the order Rhodobacterales or Hyphomonadaceae had siderophores, as no or $<1 \mathrm{~mm}$ zone of colour change was present. The Acetobacteraceae that were in closest relation to known type species of AAP, strains RB-3T and CK155 had siderophores that only chelated Fe as discussed above. In comparison, strains P40 and J01 were more genetically distant from known type species of AAP, and also produced significant zones of clearing, $\geq 10 \mathrm{~mm}$ for all metal(loid)s tested, signifying their own group. The predominantly strong expression of siderophores by AAP among the Methylobacteraceae warrant further study as most type species in this clade have not been previously recognized as phototrophs. Of note, previous research had found that isolates relating to Methylobacterium mesophilicum and M. extorquens produced siderophores, but the activity had not been linked to aerobic anoxygenic phototrophy [46]. One clade of AAP which related by 98.7-99.1\% 16S rRNA sequence similarity to Bosea lupini, including strains P13, SS335, and SS63 were all capable of strong production of metallophores, $>10 \mathrm{~mm}$ zones for multiple metal(loid)s. In comparison, strains P4 and SS56, which have $99.6 \%$ relation to $M$. phylloshaerae and $99.6 \%$ to M. branchiatum, respectively, both had stronger reactions against metal(loid)s that were not Fe (Figure A2). This activity may be explained through the findings of related works, where methanotrophs produced methanobactin, a chalkophore, which is a $\mathrm{Cu}$ specific metallophore [15]. The isolates we have tested may indeed possess similar mechanisms as they sequester $\mathrm{Cu}$ strongly, as well as $\mathrm{Mg}, \mathrm{V}, \mathrm{Mn}, \mathrm{Zn}$, and Te more favorably than Fe.

Sphingomonadaceae could be separated into 3 groups, as those related to Citromicrobium did not produce siderophores, Sphingomonas relatives produced some siderophores that were predominantly Fe specific, while relatives of Blastomonas could produce siderophores that acted on all metal(loid)s tested. A few Sphingomonas relatives had been previously found to produce siderophores against Fe [47], but not other metal(loid)s. The Blastomonas/ Erythromonas grouping was of particular interest as most representatives revealed strong metallophore production against all 10 cations tested. Our results corresponded well with previous analysis of E. ursincola, strain KR99, which had very high resistance to V, Te, and Se oxides, internally reducing them to elemental states [48]. Since KR99 can both acquire Se, $\mathrm{Te}$, and V via metallophore activity (Figure A2), and internally reduce metal(loid) oxides, it appears to require them in reduced form for some reason. Future study of such associations will determine if E. ursincola sequesters these cations as a protective measure, or uses them for some metabolic purpose. In comparison, the Erythrobacteraceae were not as concisely divided as other families, where those closest to Erythromicrobium had siderophores, but most Porphyrobacter had very small $<1 \mathrm{~mm}$ or negligible zones. An exception was strain BE100, which branched distantly from its nearest relative P. colymbi (Figure 2), and showed a significant presence of metallophores, $\sim 10 \mathrm{~mm}$ zones for all metal(loid)s except Te. Finally, strain EG19 that hailed from $\gamma$-rather than $\alpha$-Proteobacteria, had moderate siderophore production for both Fe and $\mathrm{Zn},<10 \mathrm{~mm}$. While many siderophores have been discovered as products of bacteria within the $\gamma$-proteobacterial clade (Table S1), none have been documented as highly pigmented. 


\subsection{Analysis of the Brown-Coloured Siderophore}

Gel purification of brown pigment produced by C. halotolerans (Figure 3), revealed that while $\mathrm{CBB}$ bound to proteins remained in the gel, both unbound $\mathrm{CBB}$ and brown pigment were released during destaining process. Comparing lanes $2-5$, the sample prepared by resin concentration clearly contained brown pigment, but also accumulated proteins smaller than $26 \mathrm{kDa}$. The use of the $3 \mathrm{kDa}$ cut off spin column did indeed remove these contaminants, as shown in lanes 6-8. Since the small brown pigment passed through the spin column, was purified on gel electrophoresis, and maintained activity on CAS plates, these tests confirmed that it acted as a siderophore. Furthermore, these procedures established the brown compound to clearly be under $800 \mathrm{Da}$, and approximated to be around $\sim 341$ Da when correlated to the ladder during TRIS-tricine gel electrophoresis (Figure S1). Both the estimated small size and the brown appearance of the siderophore synthesized by $C$. halotolerans were useful for its tentative identification. The most comparable small molecule described in literature was rhodotorulic acid (Table S1), a 344.4 Da siderophore that was pigmented red when bound to Fe [49]. However, this acid has only been naturally found in yeasts including Rhodotorula pilimanae, with no known bacterial producers [50]. With that in mind, hydroxamic acids are produced by both bacteria and fungi [51,52], and therefore similar secondary metabolites can be expected in other species. In addition, the colour disparity, red compared to orange-brown, may indicate an altered structure among siderophores produced by R. pilimanae and C. halotolerans, respectively.

Since $C$. halotolerans hails from the $\gamma$-Proteobacteria, comparisons must be drawn between its siderophore and those produced by other species in the $\gamma$-subclass. The most similar in size was acinetobactin, a 346.4 Da molecule from Acinetobacter baumannii expressed using the operon containing bas $A B C D E F G H I J$, bau $A B C D E F$ and bar $A B$ genes [53]. C. halotolerans genome, published within the One Thousand Microbial Genomes Phase 4 (KMG IV) project by the DOE Joint Genome Institute, submitted online in 2019 with accession number PRJNA520330 [54], contained neither similar genes nor was the operon present, while using very low homology search. Due to the divergence between $C$. halotolerans' pigmented siderophore and A. baumannii's lack of colour, and the absence of similar genes, we assume that acinetobactin was not the siderophore of $C$. halotolerans. Further structural analysis will be necessary to confirm the structural identity of this novel compound.

\section{Conclusions}

We have discovered that many AAP produce siderophores or metallophores as diffusible secondary metabolites. Production could be related to acquisition of metal(loid)s including magnesium, vanadium, manganese, iron, cobalt, nickel, copper, zinc, selenium and tellurium, or to provide resistance to toxic metal(loid)s in environments with elevated concentrations. A correlation existed between site of isolation and production of siderophores, whereas tested freshwater AAP produced siderophores, and AAP that required $\mathrm{NaCl}$ predominantly did not. Furthermore, there could be connection between phylogeny of isolates and their ability to form siderophores, but as with many phenotypes to genotype comparisons, it did not appear as a strictly followed rule. With such considerations, siderophore production cannot be recommended as a taxonomic marker for AAP identification, as variable production types occurred. However, a potential application exists to use this phenotypic feature during taxonomic differentiation between species. Future work will hopefully identify the siderophores, and potential metallophores, produced by each AAP, and determine the total list of metal cations that can be targeted.

Supplementary Materials: The following are available online at https:/ /www.mdpi.com/article/10 .3390 / microorganisms9050959/s1, Figure S1: Determination of siderophore size, Table S1: Siderophores examples listed from largest to smallest.

Author Contributions: Conceptualization, S.B.K., E.H. and V.Y.; methodology, S.B.K., E.H. and V.Y; software, S.B.K.; validation, S.B.K., V.Y.; formal analysis, S.B.K.; investigation, S.B.K., E.H.; resources, 
V.Y.; data curation, S.B.K.; writing-original draft preparation, S.B.K., E.H.; writing-review and editing, S.B.K., V.Y.; visualization, S.B.K.; supervision, V.Y.; project administration, V.Y.; funding acquisition, V.Y. All authors have read and agreed to the published version of the manuscript.

Funding: This research was funded by an NSERC Discovery Grant and a University of Manitoba GETS grant, both held by V. Yurkov.

Institutional Review Board Statement: Not applicable.

Informed Consent Statement: Not applicable.

Data Availability Statement: Data is contained within the article.

Conflicts of Interest: The authors declare no conflict of interest.

\section{Appendix A}

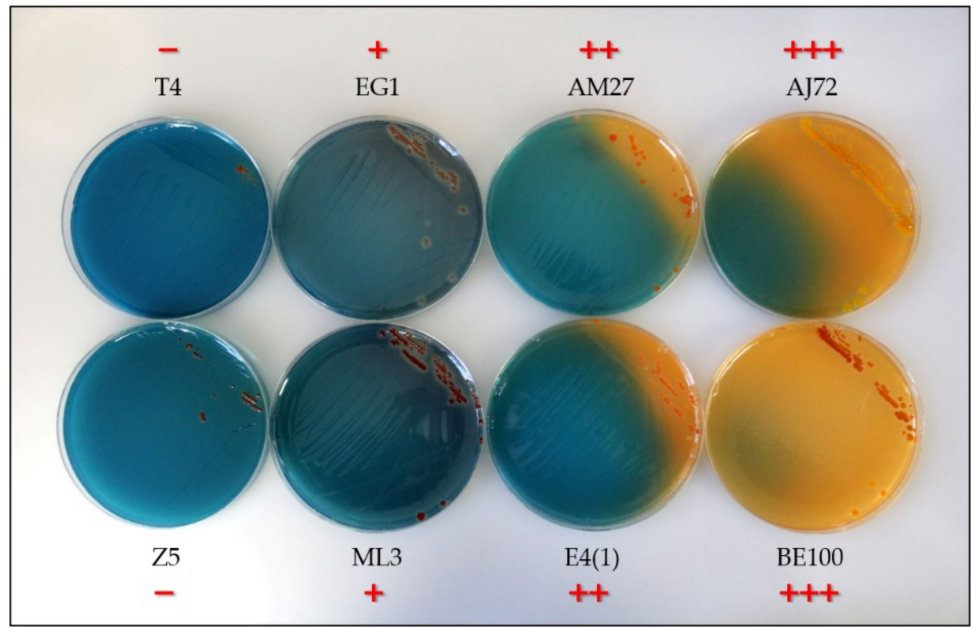

Figure A1. Iron chelating CAS assay after 5 days' growth. Zones of activity are separated into 4 groups based on range of colour change, as indicated in red by each strain name.

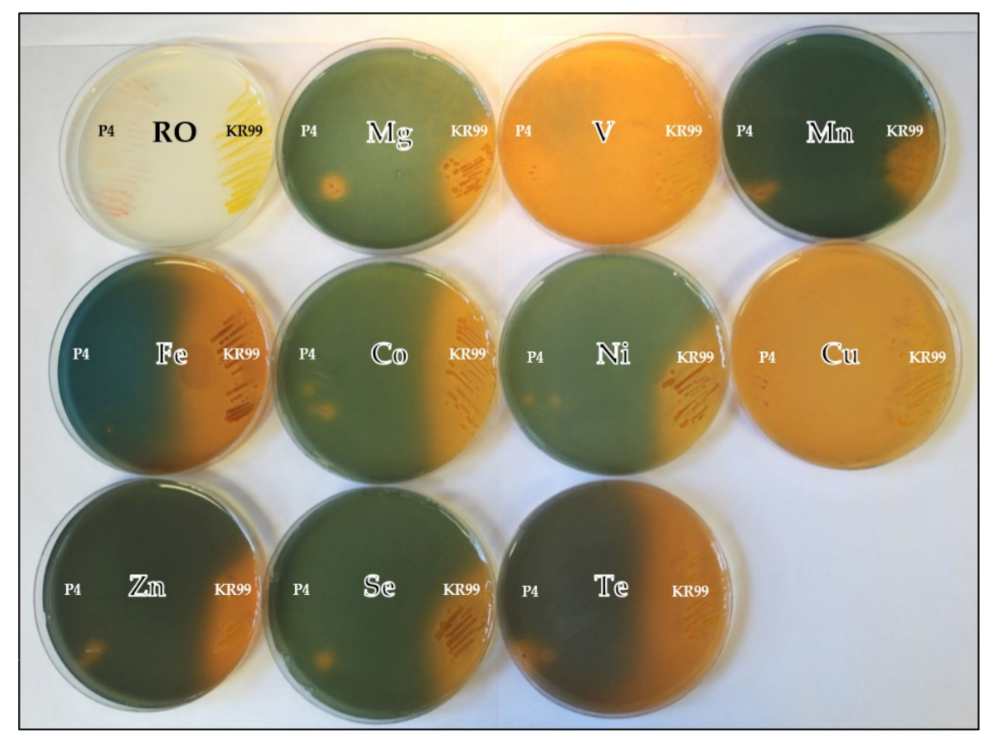

Figure A2. Variant metal(loid) CAS assay after 5 days' growth for strains P4 and KR99. Growth on $\mathrm{RO}$ without CAS was compared to those CAS plates supplemented with $\mathrm{Mg}, \mathrm{V}, \mathrm{Mn}, \mathrm{Fe}, \mathrm{Co}, \mathrm{Ni}, \mathrm{Cu}$, $\mathrm{Zn}$, Se, or Te. 
Table A1. Freshwater and saline AAP chosen for siderophore testing.

\begin{tabular}{|c|c|c|c|c|}
\hline Environment & Strain & Medium & Most Related Type Species & Accession \# \\
\hline $\begin{array}{l}\text { Hot spring, Kamchatka } \\
\text { island }\end{array}$ & $\mathrm{KR} 99^{\mathrm{T}}$ & $\mathrm{RO}$ & Erythromonas ursincola & NR_119243.1 \\
\hline \multirow{6}{*}{$\begin{array}{c}\text { Warm temperature } \\
\text { spring, Bikal Lake, } \\
\text { Russia }\end{array}$} & E1 & $\mathrm{RO}$ & 99.6\% Porphyrobacter colymbi & MW970346 \\
\hline & $\mathrm{E} 4(1)$ & $\mathrm{RO}$ & 99.7\% Porphyrobacter donghaensis & MW970347 \\
\hline & $\mathrm{E} 5^{\mathrm{T}}$ & $\mathrm{RO}$ & Erythromicrobium ramosum & NR_041891.1 \\
\hline & $\mathrm{RB}^{\mathrm{T}}$ & $\mathrm{RO}$ & Roseococcus thiosulfatophilus & NR_026114.1 \\
\hline & RB16-17 ${ }^{\mathrm{T}}$ & $\mathrm{RO}$ & Sandaracinobacter sibiricus & NR_026382.1 \\
\hline & $\mathrm{T} 4^{\mathrm{T}}$ & $\mathrm{RO}-\mathrm{NaCl}$ & Erythrobacter litoralis & NR_119016.1 \\
\hline \multirow{12}{*}{$\begin{array}{c}\text { Deep Ocean, Juan } \\
\text { DeFuco Ridge, Pacific } \\
\text { Ocean }\end{array}$} & $\mathrm{JF} 1^{\mathrm{T}}$ & $\mathrm{RO}-\mathrm{NaCl}$ & Citromicrobium bathyomarinum & Y16267.1 \\
\hline & C6 & $\mathrm{RO}-\mathrm{NaCl}$ & 98.1\% Citromicrobium bathyomarinum & MW970348 \\
\hline & $\mathrm{C} 7$ & $\mathrm{RO}-\mathrm{NaCl}$ & $99.8 \%$ Citromicrobium bathyomarinum & MW970349 \\
\hline & $\mathrm{C} 8$ & $\mathrm{RO}-\mathrm{NaCl}$ & 99.9\% Citromicrobium bathyomarinum & MW970350 \\
\hline & $\mathrm{C} 14$ & $\mathrm{RO}-\mathrm{NaCl}$ & 99.6\% Citromicrobium bathyomarinum & MW970351 \\
\hline & $\mathrm{C} 23$ & $\mathrm{RO}-\mathrm{NaCl}$ & 99.7\% Citromicrobium bathyomarinum & MW970352 \\
\hline & $\mathrm{C} 26$ & $\mathrm{RO}-\mathrm{NaCl}$ & $99.8 \%$ Citromicrobium bathyomarinum & MW970353 \\
\hline & N25 & $\mathrm{RO}-\mathrm{NaCl}$ & $99.7 \%$ Citromicrobium bathyomarinum & MW970354 \\
\hline & N34 & $\mathrm{RO}-\mathrm{NaCl}$ & 99.7\% Citromicrobium bathyomarinum & MW970355 \\
\hline & N48 & $\mathrm{RO}-\mathrm{NaCl}$ & $99.8 \%$ Citromicrobium bathyomarinum & MW970356 \\
\hline & N56 & $\mathrm{RO}-\mathrm{NaCl}$ & 99.9\% Citromicrobium bathyomarinum & MW970357 \\
\hline & N78 & $\mathrm{RO}-\mathrm{NaCl}$ & 99.6\% Citromicrobium bathyomarinum & MW970358 \\
\hline \multirow{2}{*}{$\begin{array}{l}\text { Rag Beach sediment, } \\
\text { BC, Canada }\end{array}$} & $23-\mathrm{SB}$ & $\mathrm{RO}-\mathrm{NaCl}$ & $99.7 \%$ Porphyrobacter donghaensis & MW970359 \\
\hline & $15-\mathrm{SB}$ & $\mathrm{RO}-\mathrm{NaCl}$ & $99.8 \%$ Porphyrobacter donghaensis & MW970360 \\
\hline \multirow{5}{*}{$\begin{array}{l}\text { Central Gold Mine, MB, } \\
\text { Canada }\end{array}$} & $\mathrm{C} 4$ & $\mathrm{RO}$ & 99.4\% Porphyrobacter colymbi & KX148515 \\
\hline & C9 & $\mathrm{RO}$ & $99.1 \%$ Brevundimonas variabilis & KX148516 \\
\hline & C11 & $\mathrm{RO}$ & $98.6 \%$ Brevundimonas bacteroides & KX148517 \\
\hline & NM4.16 & $\mathrm{RO}$ & $99.7 \%$ Porphyrobacter colymbi & KX148518 \\
\hline & NM4.18 & $\mathrm{RO}$ & 99.3\% Blastomonas fulva & KX148519 \\
\hline \multirow{14}{*}{$\begin{array}{c}\text { Lake Winnipeg, MB, } \\
\text { Canada }\end{array}$} & AJ 72 & RO & 99.7\% Sphingorhabdus lacus & MW970361 \\
\hline & AM 19 & $\mathrm{RO}$ & 99.7\% Erythromicrobium ramosum & MW970362 \\
\hline & AM 27 & $\mathrm{RO}$ & 99.6\% Erythromicrobium ramosum & MW970363 \\
\hline & CK 182 & $\mathrm{RO}$ & $93.4 \%$ Porphyrobacter donghaensis & MW970364 \\
\hline & BL 67 & $\mathrm{RO}$ & 98.9\% Sandarakinorhabdus cyanobacteriorum & MW970365 \\
\hline & BK 61 & $\mathrm{RO}$ & 99.1\% Blastomonas fulva & MW970366 \\
\hline & BE 100 & $\mathrm{RO}$ & $99.4 \%$ Porphyrobacter colymbi & MW970367 \\
\hline & AM 91 & $\mathrm{RO}$ & 98.5\% Porphyrobacter sanguineus & MW970368 \\
\hline & CK 155 & $\mathrm{RO}$ & 98.1\% Roseomonas sediminicola & MW970369 \\
\hline & CL 63 & $\mathrm{RO}$ & $100 \%$ Methylorubrum extorquens & MW970370 \\
\hline & BA 23 & $\mathrm{RO}$ & $96.2 \%$ Methylobacterium indicum & MW970371 \\
\hline & BC100 & $\mathrm{RO}$ & $96.6 \%$ Sphingomonas yantingensis & MW970372 \\
\hline & CN8 & $\mathrm{RO}$ & $99.2 \%$ Porphyrobacter neustonensis & MW970373 \\
\hline & GM14 & $\mathrm{RO}$ & 99.2\% Erythromonas ursincola & MW970374 \\
\hline \multirow{10}{*}{$\begin{array}{c}\text { Zebra mussels, Lake } \\
\text { Winnipeg, MB, Canada }\end{array}$} & Z1 & $\mathrm{RO}$ & 99.1\% Porphyrobacter colymbi & MN987006 \\
\hline & $\mathrm{Z2}$ & $\mathrm{RO}$ & 99.4\% Porphyrobacter tepidarius & MN987007 \\
\hline & $\mathrm{Z} 5$ & $\mathrm{RO}$ & $99.6 \%$ Porphyrobacter tepidarius & MN987008 \\
\hline & Z6 & $\mathrm{RO}$ & 99.3\% Porphyrobacter neustonensis & MN987009 \\
\hline & $\mathrm{Z7}$ & $\mathrm{RO}$ & 99.6\% Erythromicrobium ramosum & MN987010 \\
\hline & $\mathrm{Z} 24$ & $\mathrm{RO}$ & 98.7\% Porphyrobacter sanguineus & MN987011 \\
\hline & $\mathrm{Z} 27$ & $\mathrm{RO}$ & $99.4 \%$ Porphyrobacter neustonensis & MN987012 \\
\hline & Z39 & $\mathrm{RO}$ & 98.9\% Porphyrobacter sanguineus & MN987013 \\
\hline & Z59 & $\mathrm{RO}$ & 99.5\% Porphyrobacter neustonensis & MN987014 \\
\hline & Z68 & $\mathrm{RO}$ & 99.4\% Porphyrobacter neustonensis & MN987015 \\
\hline
\end{tabular}


Table A2. Meromictic lake and biological soil crust AAP chosen for siderophore testing.

\begin{tabular}{|c|c|c|c|c|}
\hline Environment & Strain & Medium & Most Related Type Species & Accession \# \\
\hline \multirow{16}{*}{$\begin{array}{c}\text { Mahoney Lake, BC, } \\
\text { Canada }\end{array}$} & ML1 & N1 & 98.4\% Erythromicrobium ramosum & MW970375 \\
\hline & ML3 & N1 & $99.4 \%$ Porphyrobacter sanguineus & MW970376 \\
\hline & $\mathrm{ML}^{\mathrm{T}}$ & N1 & Porphyrobacter meromictius & NR_115007.1 \\
\hline & $\mathrm{ML6}^{\mathrm{T}}$ & N1 & Roseicyclus mahoneyensis & NR_042080.1 \\
\hline & ML10 & N1 & $97.9 \%$ Salinarimonas ramus & MW970377 \\
\hline & ML14 & N1 & 98.9\% Erythromicrobium ramosum & MW970378 \\
\hline & ML19 & N1 & $96.9 \%$ Porphyrobacter meromiticus & MW970379 \\
\hline & ML20 & N1 & 99.3\% Blastomonas fulva & MW970380 \\
\hline & ML21 & N1 & 98.5\% Erythromicrobium ramosum & MW970381 \\
\hline & ML22 & N1 & $99.8 \%$ Porphyrobacter sanguineus & MW970382 \\
\hline & ML30 & N1 & 98.8\% Seohaeicola saemankumensis & MW970383 \\
\hline & ML35 & N1 & 99.4\% Blastomonas fulva & MW970384 \\
\hline & ML36 & N1 & 97.1\% Porphyrobacter sanguineus & MW970385 \\
\hline & ML37 & N1 & $95.4 \%$ Glycocaulis profundi & MW970386 \\
\hline & ML42 & N1 & $98.0 \%$ Roseicyclus marinus & MW970387 \\
\hline & ML45 & N1 & 94.7\% Ruegeria intermedia & MW970388 \\
\hline \multirow{10}{*}{$\begin{array}{l}\text { Blue Lake, BC, } \\
\text { Canada }\end{array}$} & BL1 & BLM & 98.1\% Roseicyclus marinus & MW970389 \\
\hline & BL4 & BLM & 99.3\% Porphyrobacter sanguineus & MW970390 \\
\hline & BL5 & BLM & $99.2 \%$ Seohaeicola saemankumensis & MW970391 \\
\hline & BL7 & BLM & $95.6 \%$ Glycocaulis profundi & MW970392 \\
\hline & BL8 & BLM & $98.1 \%$ Roseicyclus marinus & MW970393 \\
\hline & BL9 & BLM & $98.0 \%$ Roseicyclus marinus & MW970394 \\
\hline & BL11 & BLM & $95.8 \%$ Glycocaulis profundi & MW970395 \\
\hline & BL14 & BLM & $95.6 \%$ Glycocaulis profundi & MW970396 \\
\hline & BL17 & BLM & $95.1 \%$ Glycocaulis profundi & MW970397 \\
\hline & BL22 & BLM & $95.0 \%$ Glycocaulis profundi & MW970398 \\
\hline \multirow{15}{*}{$\begin{array}{c}\text { East German Creek } \\
\text { System, MB, } \\
\text { Canada }\end{array}$} & EG1 & MA & $95.2 \%$ Roseovarius pacificus & AM691094 \\
\hline & EG2 & MA & $94.4 \%$ Roseovarius bejariae & AM691093 \\
\hline & EG3 & MA & 97.3\% Yoonia vestfoldensis & AM691092 \\
\hline & EG4 & MA & 98.8\% Erythrobacter longus & AM691105 \\
\hline & EG5 & MA & 95.7\% Roseovarius pacificus & AM691095 \\
\hline & EG6 & MA & 98.4\% Porphyrobacter meromictius & AM691106 \\
\hline & EG7 & MA & 97.3\% Roseovarius nitratireducens & AM691097 \\
\hline & EG8 & MA & $99.0 \%$ Roseovarius tolerans & AM691101 \\
\hline & EG9 & MA & 97.3\% Roseovarius nitratireducens & AM691098 \\
\hline & EG10 & MA & 98.2\% Roseovarius tibetensis & AM691100 \\
\hline & EG11 & MA & 97.7\% Roseovarius nitratireducens & AM691099 \\
\hline & EG13 & MA & $99.0 \%$ Roseovarius tolerans & AM691102 \\
\hline & EG15 & MA & 98.9\% Erythrobacter aquimaris & AM691107 \\
\hline & EG17 ${ }^{\mathrm{T}}$ & MA & Charonomicrobium ambiphototrophicum & AM691091 \\
\hline & EG19 ${ }^{\mathrm{T}}$ & MA & Chromocurous halotolerans & AM691088 \\
\hline \multirow{3}{*}{$\begin{array}{c}\text { Sandy Lands Forest } \\
\text { Soil Crust, MB, } \\
\text { Canada }\end{array}$} & SS56 & BSCA & 99.6\% Methylobacterium brachiatum & MW970399 \\
\hline & SS63 & BSCA & $99.1 \%$ Bosea lupini & MW970400 \\
\hline & SS335 & BSCA & 98.3\% Bosea lupini & MW970401 \\
\hline \multirow{7}{*}{$\begin{array}{l}\text { Spruce Woods } \\
\text { National Park Soil } \\
\text { Crust, MB, Canada }\end{array}$} & J01 & BSCA & 98.4\% Belnapia moabensis & MW970402 \\
\hline & J05 & BSCA & $96.6 \%$ Sphingomonas pruni & MW970403 \\
\hline & $\mathrm{P} 4$ & BSCB & 99.6\% Methylobacterium phyllosphaerae & MW970404 \\
\hline & P13 & BSCB & 98.7\% Bosea lupini & MW970405 \\
\hline & $\mathrm{P} 40$ & BSCB & 99.9\% Belnapia soli & MW970406 \\
\hline & P132 & BSCB & 99.6\% Methylobacterium brachiatum & MW970407 \\
\hline & P233 & BSCB & 99.8\% Methylobacterium tardum & MW970408 \\
\hline
\end{tabular}




\section{References}

1. Granger, J.; Price, N.M. The importance of siderophores in iron nutrition of heterotrophic marine bacteria. Limnol. Oceanogr. 1999, 44, 541-555. [CrossRef]

2. Challis, G.L. A widely distributed bacterial pathway for siderophore biosynthesis independent of nonribosomal peptide synthetases. ChemBioChem 2005, 6, 601-611. [CrossRef] [PubMed]

3. Schalk, I.J.; Hannauer, M.; Braud, A. New roles for bacterial siderophores in metal transport and tolerance. Environ. Microbiol. 2011, 13, 2844-2854. [CrossRef] [PubMed]

4. Yurkov, V.V.; van Gemerden, H. Impact of light/dark regimen on growth rate, biomass formation and bacteriochlorophyll synthesis in Erythromicrobium hydrolyticum. Arch. Microbiol. 1993, 159, 84-89. [CrossRef]

5. Yurkov, V.; Hughes, E. Aerobic Anoxygenic Phototrophs: Four Decades of Mystery. In Modern Topics in the Phototrophic Prokaryotes: Environmental and Applied Aspects; Hallenbeck, P.C., Ed.; Springer: Cham, Switzerland, 2017; pp. 193-217.

6. Faraldo-Gómez, J.D.; Sansom, M.S.P. Acquisition of siderophores in gram-negative bacteria. Nat. Rev. Mol. Cell Biol. 2003, 4, 105-116. [CrossRef]

7. Lankford, C.E. Bacterial assimilation of iron. Crit. Rev. Microbiol. 1973, 2, 273-331. [CrossRef]

8. Reid, R.T.; Livet, D.H.; Faulkner, D.J.; Butler, A. A siderophore from a marine bacterium with an exceptional ferric ion affinity constant. Nature 1993, 366, 455-458. [CrossRef]

9. Wandersman, C.; Delepelaire, P. Bacterial iron sources: From siderophores to hemophores. Annu. Rev. Microbiol. 2004, 58, 611-647. [CrossRef]

10. Grobelak, A.; Hiller, J. Bacterial siderophores promote plant growth: Screening of catechol and hydroxamate siderophores. Int. J. Phytoremed. 2017, 19, 825-833. [CrossRef]

11. Crosa, J.H.; Walsh, C.T. Genetics and Assembly Line Enzymology of Siderophore Biosynthesis in Bacteria. Microbiol. Mol. Biol. Rev. 2002, 66, 223-249. [CrossRef]

12. Lamont, I.L.; Beare, P.A.; Ochsner, U.; Vasil, A.I.; Vasil, M.L. Siderophore-mediated signaling regulates virulence factor production in Pseudomonas aeruginosa. Proc. Natl. Acad. Sci. USA 2002, 99, 7072-7077. [CrossRef]

13. Adler, C.; Corbalán, N.S.; Seyedsayamdost, M.R.; Pomares, M.F.; de Cristóbal, R.E.; Clardy, J.; Kolter, R.; Vincent, P.A. Catecholate Siderophores Protect Bacteria from Pyochelin Toxicity. PLoS ONE 2012, 7, e46754. [CrossRef]

14. Ghssein, G.; Brutesco, C.; Ouerdane, L.; Fojcik, C.; Izaute, A.; Wang, S.; Hajjar, C.; Lobinski, R.; Lemaire, D.; Richaud, P.; et al. Biosynthesis of a broad-spectrum nicotianamine-like metallophore in Staphylococcus aureus. Science 2016, 352, 1105-1109. [CrossRef]

15. Kim, H.J.; Graham, D.W.; DiSpirito, A.A.; Alterman, M.A.; Galeva, N.; Larive, C.K.; Asunskis, D.; Sherwood, P.M.A. Methanobactin, a copper-acquisition compound from methane-oxidizing bacteria. Science 2004, 305, 1612-1615. [CrossRef]

16. Morey, J.R.; Kehl-Fie, T.E. Bioinformatic Mapping of Opine-Like Zincophore Biosynthesis in Bacteria. mSystems 2020, 5, 1-16. [CrossRef]

17. Welch, R.M.; Shuman, L. Micronutrient Nutrition of Plants. CRC Crit. Rev. Plant Sci. 1995, 14, 49-82. [CrossRef]

18. Pedler, J.F.; Parker, D.R.; Crowley, D.E. Zinc deficiency-induced phytosiderophore release by the Triticaceae is not consistently expressed in solution culture. Planta 2000, 211, 120-126. [CrossRef]

19. Nies, D.H. Heavy metal-resistant bacteria as extremophiles: Molecular physiology and biotechnological use of Ralstonia sp. CH34. Extremophiles 2000, 4, 77-82. [CrossRef]

20. Rothschild, L.J.; Mancinelli, R.L. Life in extreme environments. Nature 2001, 409, 1092-1101. [CrossRef]

21. Csotonyi, J.T.; Maltman, C.; Yurkov, V. Influence of tellurite on synthesis of bacteriochlorophyll and carotenoids in aerobic anoxygenic phototrophic bacteria. Res. Trends Photochem. Photobiol. 2014, 16, 1-17.

22. Maltman, C.; Yurkov, V. The Effect of Tellurite on Highly Resistant Freshwater Aerobic Anoxygenic Phototrophs and Their Strategies for Reduction. Microorganisms 2015, 3, 826-838. [CrossRef]

23. Csotonyi, J.T.; Swiderski, J.; Stackebrandt, E.; Yurkov, V.V. Novel halophilic aerobic anoxygenic phototrophs from a Canadian hypersaline spring system. Extremophiles 2008, 12, 529-539. [CrossRef]

24. Csotonyi, J.T.; Stackebrandt, E.; Swiderski, J.; Schumann, P.; Yurkov, V. Chromocurvus halotolerans gen. nov., sp. nov., a gammaproteobacterial obligately aerobic anoxygenic phototroph, isolated from a Canadian hypersaline spring. Arch. Microbiol. 2011, 193, 573-582. [CrossRef]

25. Drechsel, H.; Jung, G. Peptide siderophores. J. Pept. Sci. 1998, 4, 147-181. [CrossRef]

26. Yurkov, V. Aerobic Phototrophic Proteobacteria. In The Prokaryotes; Dworkin, M., Falkow, S., Rosenberg, E., Schleifer, K.-H., Stackebrandt, E., Eds.; Springer: New York, NY, USA, 2006; pp. 562-584.

27. Csotonyi, J.T.; Swiderski, J.; Stackebrandt, E.; Yurkov, V. A new environment for aerobic anoxygenic phototrophic bacteria: Biological soil crusts. Environ. Microbiol. Rep. 2010, 2, 651-656. [CrossRef]

28. Yurkova, N.; Rathgeber, C.; Swiderski, J.; Stackebrandt, E.; Beatty, J.T.; Hall, K.J.; Yurkov, V. Diversity, distribution and physiology of the aerobic phototrophic bacteria in the mixolimnion of a meromictic lake. FEMS Microbiol. Ecol. 2002, 40, 191-204. [CrossRef]

29. Kuzyk, S.B.; Pritchard, A.O.; Plouffe, J.; Sorensen, J.L.; Yurkov, V. Psychrotrophic violacein-producing bacteria isolated from Lake Winnipeg, Canada. J. Great Lakes Res. 2020. [CrossRef]

30. Kuzyk, S.B.; Wiens, K.; Ma, X.; Yurkov, V. Association of aerobic anoxygenic phototrophs and zebra mussels, Dreissena polymorpha, within the littoral zone of Lake Winnipeg. J. Great Lakes Res. 2020. [CrossRef] 
31. Schwyn, B.; Neilands, J.B. Universal chemical assay for the detection and determination of siderophores. Anal. Biochem. 1987, 160, 47-56. [CrossRef]

32. Rainey, F.A.; Ward-Rainey, N.; Kroppenstedt, R.M.; Stackebrandt, E. The genus Nocardiopsis represents a phylogenetically coherent taxon and a distinct actinomycete lineage: Proposal of Nocardiopsaceae fam. nov. Int. J. Syst. Bacteriol. 1996, 46, 1088-1092. [CrossRef]

33. Sanger, F.; Coulson, A.R. A rapid method for determining sequences in DNA by primed synthesis with DNA polymerase. J. Mol. Biol. 1975, 94, 441-448. [CrossRef]

34. Madden, T. The BLAST Sequence Analysis Tool. In The NCBI Handbook [Internet]; McEntyre, J., Ostell, J., Eds.; National Center for Biotechnology Information (US): Bethesda, MD, USA, 2002; pp. 1-15.

35. Kumar, S.; Stecher, G.; Li, M.; Knyaz, C.; Tamura, K. MEGA X: Molecular evolutionary genetics analysis across computing platforms. Mol. Biol. Evol. 2018, 35, 1547-1549. [CrossRef] [PubMed]

36. Felsenstein, J. Confidence Limits on Phylogenies: An Approach Using the Bootstrap. Evolution 1985, 39, 783-791. [CrossRef] [PubMed]

37. Nei, M.; Kumar, S. Molecular Evolution and Phylogenetics; Oxford University Press: New York, NY, USA, 2000.

38. Barker, R.; Boden, N.; Cayley, G.; Charlton, S.C.; Henson, R.; Holmes, M.C.; Kelly, I.D.; Knowles, P.F. Properties of cupric ions in benzylamine oxidase from pig plasma as studied by magnetic-resonance and kinetic methods. Biochem. J. 1979, 177, $289-302$. [CrossRef]

39. Yamamoto, S.; Okujo, N.; Sakakibara, Y. Isolation and structure elucidation of acinetobactin., a novel siderophore from Acinetobacter baumannii. Arch. Microbiol. 1994, 162, 249-254.

40. Schägger, H.; von Jagow, G. Tricine-sodium dodecyl sulfate-polyacrylamide gel electrophoresis for the separation of proteins in the range from 1 to $100 \mathrm{kDa}$. Anal. Biochem. 1987, 166, 368-379. [CrossRef]

41. Arora, N.K.; Verma, M. Modified microplate method for rapid and efficient estimation of siderophore produced by bacteria. 3 Biotech 2017, 7, 1-9. [CrossRef]

42. Ames-Gottfred, N.P.; Christie, B.R.; Jordan, D.C. Use of the Chrome Azurol S Agar Plate Technique to Differentiate Strains and Field Isolates of Rhizobium leguminosarum biovar trifolii. Appl. Environ. Microbiol. 1989, 55, 707-710. [CrossRef]

43. Amaro, C.; Aznar, R.; Alcaide, E.; Lemos, M.L. Iron-binding compounds and related outer membrane proteins in Vibrio cholerae non-O1 strains from aquatic environments. Appl. Environ. Microbiol. 1990, 56, 2410-2416. [CrossRef]

44. Patel, P.R.; Shaikh, S.S.; Sayyed, R.Z. Modified chrome azurol S method for detection and estimation of siderophores having affinity for metal ions other than iron. Environ. Sustain. 2018, 1, 81-87. [CrossRef]

45. Rensing, C.; Ghosh, M.; Rosen, B.P. Families of Soft-Metal-Ion-Transporting ATPases. J. Bacteriol. 1999, 181, 5891-5897. [CrossRef]

46. Idris, R.; Trifonova, R.; Puschenreiter, M.; Wenzel, W.W.; Sessitsch, A. Bacterial communities associated with flowering plants of the Ni hyperaccumulator Thlaspi goesingense. Appl. Environ. Microbiol. 2004, 70, 2667-2677. [CrossRef]

47. Sun, L.N.; Zhang, Y.F.; He, L.Y.; Chen, Z.J.; Wang, Q.Y.; Qian, M.; Sheng, X.F. Genetic diversity and characterization of heavy metal-resistant-endophytic bacteria from two copper-tolerant plant species on copper mine wasteland. Bioresour. Technol. 2010, 101, 501-509. [CrossRef]

48. Maltman, C.; Donald, L.; Yurkov, V. Tellurite and Tellurate Reduction by the Aerobic Anoxygenic Phototroph Erythromonas ursincola, Strain KR99 Is Carried out by a Novel Membrane Associated Enzyme. Microorganisms 2017, 5, 20. [CrossRef]

49. Atkin, C.L.; Neilands, J.B. Rhodotorulic Acid, a Diketopiperazine Dihydroxamic Acid with Growth-Factor Activity. I. Isolation and Characterization. Biochemistry 1968, 7, 3734-3739. [CrossRef]

50. Andersen, D.; Renshaw, J.C.; Wiebe, M.G. Rhodotorulic acid production by Rhodotorula mucilaginosa. Mycol. Res. 2003, 107, 949-956. [CrossRef]

51. Carson, K.C.; Meyer, J.M.; Dilworth, M.J. Hydroxamate siderophores of root nodule bacteria. Soil Biol. Biochem. 2000, 32, 11-21. [CrossRef]

52. Holinsworth, B.; Martin, J.D. Siderophore production by marine-derived fungi. BioMetals 2009, 22, 625-632. [CrossRef]

53. Mihara, K.; Tanabe, T.; Yamakawa, Y.; Funahashi, T.; Nakao, H.; Narimatsu, S.; Yamamoto, S. Identification and transcriptional organization of a gene cluster involved in biosynthesis and transport of acinetobactin, a siderophore produced by Acinetobacter baumannii ATCC 19606T. Microbiology 2004, 150, 2587-2597. [CrossRef]

54. Goeker, M. The One Thousand Microbial Genomes Phase 4 Project (KMG-4) Sequencing the Most Valuable Type-Strain Genomes for Metagenomic Binning, Comparative Biology and Taxonomic Classification; DOE Joint Genome Institute: Oak Ridge, TN, USA, 2016. [CrossRef] 